Pacific

Journal of

Mathematics

CLASSIFICATION OF POSITIVE SOLUTIONS

FOR AN ELLIPTIC SYSTEM

WITH A HIGHER-ORDER FRACTIONAL LAPLACIAN

JingBo Dou AND ChANGZHeng QU 


\title{
CLASSIFICATION OF POSITIVE SOLUTIONS FOR AN ELLIPTIC SYSTEM WITH A HIGHER-ORDER FRACTIONAL LAPLACIAN
}

\author{
JingBo Dou AND ChANGZHENG QU
}

We discuss properties of solutions to the following elliptic PDE system in $\mathbb{R}^{n}$ :

$$
\left\{\begin{array}{l}
(-\Delta)^{\alpha / 2} u=\lambda_{1} u^{p_{1}}+\mu_{1} v^{p_{2}}+\beta_{1} u^{p_{3}} v^{p_{4}}, \\
(-\Delta)^{\alpha / 2} v=\lambda_{2} u^{q_{1}}+\mu_{2} v^{q_{2}}+\beta_{2} u^{q_{3}} v^{q_{4}},
\end{array}\right.
$$

where $0<\alpha<n, \lambda_{j}, \mu_{j}, \beta_{j}(j=1,2)$ are nonnegative constants and $p_{i}$ and $q_{i}(i=1,2,3,4)$ satisfy some suitable assumptions. It is shown that this PDE system is equivalent to the integral system

$$
\left\{\begin{array}{l}
u(x)=\int_{\mathbb{R}^{n}} \frac{\lambda_{1} u^{p_{1}}(y)+\mu_{1} v^{p_{2}}(y)+\beta_{1} u^{p_{3}}(y) v^{p_{4}}(y)}{|x-y|^{n-\alpha}} d y, \\
v(x)=\int_{\mathbb{R}^{n}} \frac{\lambda_{2} u^{q_{1}}(y)+\mu_{2} v^{q_{2}}(y)+\beta_{2} u^{q_{3}}(y) v^{q_{4}}(y)}{|x-y|^{n-\alpha}} d y
\end{array}\right.
$$

in $\mathbb{R}^{n}$. The radial symmetry, monotonicity and regularity of positive solutions are proved via the method of moving plane in integral forms and a regularity lifting lemma. For the special case with

$$
p_{1}=p_{2}=q_{1}=q_{2}=p_{3}+p_{4}=q_{3}+q_{4}=\frac{n+\alpha}{n-\alpha},
$$

positive solutions of the integral system (or the PDE system) are classified. Furthermore, our symmetry results, together with some known results on nonexistence of positive solutions, imply that, under certain integrability conditions, the PDE system has no positive solution in the subcritical case.

\section{Introduction}

In this paper, we study positive solutions of the following higher-order elliptic system in $\mathbb{R}^{n}$ :

$$
\left\{\begin{array}{l}
(-\Delta)^{\alpha / 2} u=\lambda_{1} u^{p_{1}}+\mu_{1} v^{p_{2}}+\beta_{1} u^{p_{3}} v^{p_{4}} \\
(-\Delta)^{\alpha / 2} v=\lambda_{2} u^{q_{1}}+\mu_{2} v^{q_{2}}+\beta_{2} u^{q_{3}} v^{q_{4}}
\end{array}\right.
$$

MSC2010: 35J99, 45K05, 35R09, 35J48, 35B65, 45G15.

Keywords: system of integral equations, regularity, moving plane method in integral form, classification of solutions. 
where $(-\Delta)^{\alpha / 2}$ is a higher-order fractional Laplacian, $0<\alpha<n, \lambda_{i}, \mu_{i}, \beta_{i} \geq 0$ $(i=1,2)$ are constants, and $p_{i}$ and $q_{i}(i=1,2,3,4)$ satisfy some suitable assumptions.

System (1) arises from $N$-coupled higher-order nonlinear Schrödinger systems

$$
\left\{\begin{array}{l}
i \frac{\partial \Phi_{j}}{\partial t}-(-\Delta)^{m} \Phi_{j}+\sum_{i=1}^{N} \beta_{i j}\left|\Phi_{i}\right|^{2} \Phi_{j}=0, \quad y \in \mathbb{R}^{n}, t>0, \\
\Phi_{j}(y, t) \rightarrow 0, \quad \text { as } y \rightarrow \infty, t>0, j=1,2, \ldots, N,
\end{array}\right.
$$

for $m \in \mathbb{N}, \beta_{i j}=\beta_{j i}$. System (2) appears in some physical problems, especially in nonlinear optics. When $m=1, n \leq 3$, it describes physical phenomena such as the propagation in birefringent optical fibers, Kerr-like photo refractive media in optics (see [Akhmediev and Ankiewicz 1999]) and Bose-Einstein condensates (see [Esry et al. 1997]). When the spatial dimension is one, i.e., $n=1$, system (2) has applications in quantum mechanics (see [Liu et al. 2007; Fu et al. 2009]).

Letting $\Phi_{j}(y, t)=e^{-i \lambda_{j} t} u_{j}(y)$, system (2) is transformed into the elliptic system

$$
\begin{cases}(-\Delta)^{m} u_{j}=\lambda_{j} u_{j}+\sum_{i=1}^{N} \beta_{i j}\left|u_{i}\right|^{2} u_{j} & \text { in } \mathbb{R}^{n}, \\ u_{j}(x) \rightarrow 0, & \text { as }|x| \rightarrow \infty, j=1,2, \ldots, N .\end{cases}
$$

Clearly, in some sense, system (1) extends system (3) with $N=2$.

For further discussion, we need an integral form of system (1). In Section 5, we will show that system (1) is equivalent to the following system:

$$
\left\{\begin{array}{l}
u(x)=\int_{\mathbb{R}^{n}} \frac{\lambda_{1} u^{p_{1}}(y)+\mu_{1} v^{p_{2}}(y)+\beta_{1} u^{p_{3}}(y) v^{p_{4}}(y)}{|x-y|^{n-\alpha}} d y, \\
v(x)=\int_{\mathbb{R}^{n}} \frac{\lambda_{2} u^{q_{1}}(y)+\mu_{2} v^{q_{2}}(y)+\beta_{2} u^{q_{3}}(y) v^{q_{4}}(y)}{|x-y|^{n-\alpha}} d y .
\end{array}\right.
$$

In particular, when $\mu_{1}=\lambda_{2}=1$ and $\lambda_{1}=\mu_{2}=\beta_{1}=\beta_{2}=0$, system (4) reduces to

$$
\left\{\begin{array}{l}
u(x)=\int_{\mathbb{R}^{n}} \frac{v^{p_{2}}(y)}{|x-y|^{n-\alpha}} d y, \\
v(x)=\int_{\mathbb{R}^{n}} \frac{u^{q_{1}}(y)}{|x-y|^{n-\alpha}} d y,
\end{array}\right.
$$

which is closely related to the maximizer of the best constant in a Hardy-LittlewoodSobolev (HLS) inequality; see [Chen et al. 2005; Chen and Li 2005].

In recent years many works have been devoted to the study of the special cases of system (1) or system (4). In the case of $\alpha=2$, under certain assumptions, the existence of bound state solutions and radially symmetric solutions of (3) was studied in [Bartsch et al. 2007; 2010; Busca and Sirakov 2000; Dancer et al. 2010; Liu and Wang 2008; Guo and Liu 2008; Hioe 1999; Lin and Wei 2005; Maia 
et al. 2006; Sirakov 2007; Wei and Weth 2007; 2008]. In particular, for $\alpha=2$ $(n \geq 3)$ and $\lambda_{i}=\mu_{i}=1, \beta_{i} \geq 0(i=1,2)$, de Figueiredo and Sirakov [2005] proved the nonexistence of positive solutions for system (1) under some subcritical exponent conditions. When $m=n=1$, system (2) is integrable, and there are many analytical and numerical results on solitary wave solutions of higher-order nonlinear Schrödinger equations (e.g., see [Liu et al. 2007; Fu et al. 2009]).

In the case of $\alpha=2 m(m=1,2, \ldots)$ and $\mu_{1}=\lambda_{2}=1, \lambda_{1}=\mu_{2}=\beta_{1}=\beta_{2}=0$, system (1) becomes

$$
\left\{\begin{array}{l}
(-\Delta)^{m} u=v^{p_{2}} \\
(-\Delta)^{m} v=u^{q_{1}}
\end{array}\right.
$$

in $\mathbb{R}^{n}$. This system is equivalent to the integral system (5) with $\alpha=2 m$ (see [Chen and Li 2009b]). Guo, Liu and Zhang [Liu et al. 2006; Zhang 2007] proved that any positive solutions of (6) are radially symmetric for critical exponents $p_{2}=q_{1}=\frac{n+2 m}{n-2 m}$. Moreover, they also showed that there are no positive solutions of (6) if $p_{2}, q_{1} \geq 1$, but are not both equal to 1 , and satisfy the following subcritical exponent condition:

$$
\frac{1}{p_{2}+1}+\frac{1}{q_{1}+1}>\frac{n-2 m}{n} \text {. }
$$

Assuming that $p_{2}$ and $q_{1}$ satisfy $\frac{\alpha}{n-\alpha}<p_{2}, q_{1}<\infty$, under natural integrability conditions on $u$ and $v$, Chen, Li and $\mathrm{Ou}$ [Chen et al. 2005; Chen and Li 2005] and Hang [2007] discussed the symmetry, monotonicity and regularity of positive solutions of system (5) with the critical exponent condition

$$
\frac{1}{p_{2}+1}+\frac{1}{q_{1}+1}=\frac{n-\alpha}{n} \text {. }
$$

Furthermore, Chen and Li [2009b] proved the nonexistence of positive solutions of system (5) satisfying some subcritical exponents assumptions.

In [Dou et al. 2011], we studied the symmetry, monotonicity and regularity of positive solutions of integral system (5) with weighted functions for $\max \left\{1, \frac{\alpha}{n-\alpha}\right\}<$ $p_{2}, q_{1}<\infty$ and

$$
\frac{1}{p_{2}+1}+\frac{1}{q_{1}+1} \geq \frac{n-\alpha}{n} .
$$

In addition, the nonexistence result for positive solutions of system (5) with $0<$ $p_{2}, q_{1}<\frac{n+\alpha}{n-\alpha}$ was established.

In the case of $\lambda_{i}=1, \mu_{i}=\beta_{i}=0$ and $u(x)=v(x)$, system (1) reduces to the single elliptic equation

$$
(-\Delta)^{\alpha / 2} u=u^{p}, \quad \text { in } \mathbb{R}^{n} .
$$

For $p=\frac{n+\alpha}{n-\alpha}$, Chen et al. [2006] and Li [2004] proved that any positive solutions $u$ of Equation (7) are radially symmetric and monotonic about some point. Indeed all 
the positive solutions are given by

$$
u(x)=\left(\frac{C_{\alpha}}{d+|x-\bar{x}|^{2}}\right)^{(n-\alpha) / 2},
$$

where $d>0$ is a constant and $C_{\alpha}=\left(2^{-\alpha} \Gamma\left(\frac{n+\alpha}{2}\right) \Gamma\left(\frac{n-\alpha}{2}\right)^{-1} d\right)^{1 / 2}$. When $\alpha=2 m$ is any even number, the above result was also proved by Wei and Xu [1999], and they showed that there exist no positive solutions of (7) with $0<\tau<\frac{n+2 m}{n-2 m}$. Moreover, for $\alpha=2$, the problem is the so-called Yamabe problem, and the radial symmetry of solutions was discussed by Gidas, Ni and Nirenberg [Gidas et al. 1981].

In this paper, we show that system (1) is equivalent to integral system (4). By the discussion of the symmetry, monotonicity and regularity of positive solutions of integral system (4), we are able to perform the classification of positive solutions to system (1).

Throughout the paper, we use the following notation:

$$
\begin{aligned}
& \Pi_{1}=\left\{f(x) \mid x \in \mathbb{R}^{n}, f \in L^{s_{11}}\left(\mathbb{R}^{n}\right) \cap L^{s_{21}}\left(\mathbb{R}^{n}\right) \cap L^{k_{0}}\left(\mathbb{R}^{n}\right)\right\}, \\
& \Pi_{2}=\left\{f(x) \mid x \in \mathbb{R}^{n}, f \in L^{s_{12}}\left(\mathbb{R}^{n}\right) \cap L^{s_{22}}\left(\mathbb{R}^{n}\right) \cap L^{k_{0}}\left(\mathbb{R}^{n}\right)\right\},
\end{aligned}
$$

where $s_{1 i}=n\left(p_{i}-1\right) / \alpha, s_{2 i}=n\left(q_{i}-1\right) / \alpha, i=1,2$, and $k_{0}=n\left(p_{3}+p_{4}-1\right) / \alpha=$ $n\left(q_{3}+q_{4}-1\right) / \alpha$ with $n /(n-\alpha)<p_{i}, q_{i}, p_{3}+p_{4}, q_{3}+q_{4}<\infty$, and $p_{3}+p_{4}=q_{3}+q_{4}$.

We are now in a position to state our main results.

Theorem 1.1. Assume that $\lambda_{i}, \mu_{i}, \beta_{i} \geq 0(i=1,2)$, and they are not equal to zero simultaneously. Let $(u, v)$ be a pair of solutions to system (4) with $u \in \Pi_{1}, v \in \Pi_{2}$. Then $u, v \in L^{s}\left(\mathbb{R}^{n}\right) \cap L^{\infty}\left(\mathbb{R}^{n}\right)$ for any $\frac{n}{n-\alpha}<s<\infty$. Furthermore, $u, v \in C^{\infty}$.

Theorem 1.2. Assume that $\lambda_{i}, \mu_{i}, \beta_{i} \geq 0(i=1,2)$ and they are not equal to zero at the same time. Let $(u, v) \in \Pi_{1} \times \Pi_{2}$ be a pair of solutions to system (4). Then $u$ and $v$ are radially symmetric and decreasing about some point.

For system (4) with critical exponents, i.e., $p_{1}=p_{2}=q_{1}=q_{2}=p_{3}+p_{4}=$ $q_{3}+q_{4}=\frac{n+\alpha}{n-\alpha}$, we have:

Theorem 1.3. Let $(u, v) \in L^{2 n /(n-\alpha)}\left(\mathbb{R}^{n}\right) \times L^{2 n /(n-\alpha)}\left(\mathbb{R}^{n}\right)$ be a pair of positive solutions to system (4) with $\lambda_{i}, \mu_{i}, \beta_{i} \geq 0(i=1,2)$ but not equal to zero at the same time. If $p_{1}=p_{2}=q_{1}=q_{2}=p_{3}+p_{4}=q_{3}+q_{4}=\frac{n+\alpha}{n-\alpha}$, then $u, v \in L^{s}\left(\mathbb{R}^{n}\right) \cap L^{\infty}\left(\mathbb{R}^{n}\right)$ for any $\frac{n}{n-\alpha}<s<\infty$, and $u, v \in C^{\infty}$. Moreover, $u$ and $v$ are radially symmetric and decreasing about some point, and $u, v$ must be of the following forms:

$$
u(x)=\left(\frac{c_{1}}{d+|x-\bar{x}|^{2}}\right)^{(n-\alpha) / 2}, \quad v(x)=\left(\frac{c_{2}}{d+|x-\bar{x}|^{2}}\right)^{(n-\alpha) / 2},
$$

where $\bar{x} \in \mathbb{R}^{n}, c_{1}, c_{2}>0, d>0$ and satisfy the conditions 


$$
\begin{aligned}
& C_{\alpha}^{2} c_{1}^{(n-\alpha) / 2}=\lambda_{1} c_{1}^{(n+\alpha) / 2}+\mu_{1} c_{2}^{(n+\alpha) / 2}+\beta_{1}\left(c_{1}^{p_{3}} c_{2}^{p_{4}}\right)^{(n-\alpha) / 2}, \\
& C_{\alpha}^{2} c_{2}^{(n-\alpha) / 2}=\lambda_{2} c_{1}^{(n+\alpha) / 2}+\mu_{2} c_{2}^{(n+\alpha) / 2}+\beta_{2}\left(c_{1}^{q_{3}} c_{2}^{q_{4}}\right)^{(n-\alpha) / 2} .
\end{aligned}
$$

Theorem 1.4. System (1) is equivalent to integral system (4).

Combining our symmetry and equivalence results with the known results on nonexistence of positive solutions in the subcritical case (see [Dancer et al. 2010; de Figueiredo and Sirakov 2005]), we can obtain results on the nonexistence of positive solutions $(u, v)$ of system (1) with some suitable conditions.

Theorem 1.5. (i) Suppose $n \geq 3, \alpha=2, \lambda_{i}=\mu_{i}=1$ and $\beta_{i}=0$ for $i=1,2$, and

$$
\frac{n}{n-2}<p_{1}, q_{2}<\frac{n+2}{n-2}, \quad p_{2}=\frac{p_{1}\left(q_{2}-1\right)}{p_{1}-1}, \quad q_{1}=\frac{q_{2}\left(p_{1}-1\right)}{q_{2}-1} .
$$

Then system (1) has no positive solutions $(u, v)$ satisfying $u \in L^{n\left(p_{1}-1\right) / 2}\left(\mathbb{R}^{n}\right) \cap$ $L^{n\left(q_{1}-1\right) / 2}\left(\mathbb{R}^{n}\right) \cap L^{\infty}\left(\mathbb{R}^{n}\right)$ and $v \in L^{n\left(p_{2}-1\right) / 2}\left(\mathbb{R}^{n}\right) \cap L^{n\left(q_{2}-1\right) / 2}\left(\mathbb{R}^{n}\right) \cap L^{\infty}\left(\mathbb{R}^{n}\right)$.

(ii) Assume that $n \geq 3, \alpha=2$ and $\lambda_{i}, \mu_{i}>0, \beta_{i} \geq 0, \beta_{i} \not \equiv 0$, and $p_{j}, q_{j}$ satisfy

$$
\frac{n}{n-2}<p_{1}, q_{2}<\frac{n+2}{n-2} \quad \text { with } \quad p_{2}=\frac{p_{1}\left(q_{2}-1\right)}{p_{1}-1}, \quad q_{1}=\frac{q_{2}\left(p_{1}-1\right)}{q_{2}-1}
$$

and

$$
\frac{p_{3}}{p_{1}}+\frac{p_{4}}{p_{2}}=\frac{q_{3}}{q_{1}}+\frac{q_{4}}{q_{2}}=1
$$

with $0 \leq p_{3} \leq p_{1}, 0 \leq p_{4} \leq p_{2}, 0 \leq q_{3} \leq q_{1}, 0 \leq q_{4} \leq q_{2}, p_{3}+p_{4}=q_{3}+q_{4}$. Then system (1) has no positive solutions $(u, v)$ satisfying $u \in \Pi_{1} \cap L^{\infty}\left(\mathbb{R}^{n}\right)$ and $v \in \Pi_{2} \cap L^{\infty}\left(\mathbb{R}^{n}\right)$.

(iii) Assume that $n=3, \alpha=2, \lambda_{2}=\mu_{1}=0, \beta_{1}=\beta_{2}>-\sqrt{\lambda_{1} \mu_{2}}$. Then system (1) has no positive solutions $(u, v)$ satisfying $u \in \Pi_{1} \cap L^{\infty}\left(\mathbb{R}^{n}\right)$ and $v \in \Pi_{2} \cap L^{\infty}\left(\mathbb{R}^{n}\right)$, where $p_{1}=q_{2}=3, p_{3}=q_{4}=1, p_{4}=q_{3}=2$.

Remark 1.6. We can show that the results above hold for the more general system

$$
\left\{\begin{array}{l}
(-\Delta)^{\alpha / 2} u=\lambda_{1} u^{p_{1}}+\mu_{1} v^{p_{2}}+\beta_{1} u^{p_{3}} v^{p_{4}} \\
(-\Delta)^{\kappa / 2} v=\lambda_{2} u^{q_{1}}+\mu_{2} v^{q_{2}}+\beta_{2} u^{q_{3}} v^{q_{4}}
\end{array}\right.
$$

in $\mathbb{R}^{n}$, where $0<\alpha, \kappa<n, \lambda_{i}, \mu_{i}, \beta_{i} \geq 0(i=1,2)$. That is, if

$p_{1}, p_{2}, p_{3}+p_{4}>\frac{n}{n-\alpha}, \quad q_{1}, q_{2}, q_{3}+q_{4}>\frac{n}{n-\kappa}, \quad s_{1 i}=\frac{n\left(p_{i}-1\right)}{\alpha}, \quad s_{2 i}=\frac{n\left(q_{i}-1\right)}{\kappa}$ for $i=1,2$, and $u, v \in L^{k_{0}}\left(\mathbb{R}^{n}\right) \cap L^{k_{1}}\left(\mathbb{R}^{n}\right)$, where

$$
k_{0}=\frac{n\left(p_{3}+p_{4}-1\right)}{\alpha}, \quad k_{1}=\frac{n\left(q_{3}+q_{4}-1\right)}{\kappa},
$$

then the results of Theorem 1.1, Theorem 1.2 and Theorem 1.4 are still valid. 
We remark that a more general system of $m$ equations has been discussed by Chen and Li [2009a]. That is,

$$
\left\{\begin{array}{l}
u_{j}(x)=\int_{\mathbb{R}^{n}} \frac{f_{j}(u(y))}{|x-y|^{n-\alpha}} d y, \quad j=1,2, \ldots, m, \quad \text { in } \mathbb{R}^{n} \\
u(x)=\left(u_{1}(x), u_{2}(x), \ldots, u_{m}(x)\right),
\end{array}\right.
$$

where $f_{j}(u) \geq 0$ are continuous real-valued functions and homogeneous of degree $\frac{n+\alpha}{n-\alpha}$, and satisfy $\partial f_{i} / \partial u_{j} \geq 0$ for $i=1,2, \ldots, m$. System (11) includes only the critical exponent case of system (4). It was shown in [Chen and Li 2009a] any positive solutions of (11) are radially symmetric under the assumptions $u_{j} \in$ $L_{\text {loc }}^{\infty}\left(\mathbb{R}^{n}\right)$. Furthermore, based on the Kelvin transformation and the results in [Chen et al. 2006], any positive solutions of (11) must be the form of (8). In our proof of Theorem 1.3, a key calculus lemma due to Li and Zhu [1995] and the Kelvin transformation are used to show that all positive solutions of (4) are given by (9).

The main difficulty in our proof is the lack of a maximum principle for the higherorder fractional Laplace operator. Theorem 1.4 says that system (1) is equivalent to the integral system (4), which is helpful for our discussion since we can use the method of moving planes in integral forms (see [Chen et al. 2006]) to discuss the radial symmetry and monotonicity of positive solution of the integral system (4). Furthermore, the regularity of solutions to system (4) is proved by the regularity lifting lemma introduced in [Chen and Li 2010; Ma et al. 2011].

The paper is organized as follows. In Section 2, we prove the regularity of solutions of system (4) (Theorem 1.1). The radially symmetric property and monotonicity of solutions are studied in Section 3 (Theorem 1.2). In Section 4, positive solutions of system (4) with critical exponents are classified. Namely, Theorem 1.3 is proved. In Section 5, we obtain some nonexistence results by proving Theorems 1.4 and 1.5.

Throughout the paper, we always assume that $\lambda_{i}, \mu_{i}, \beta_{i} \geq 0(i=1,2)$ and they are not equal to zero simultaneously. Moreover, for convenience of presentation we shall use $c, c_{1}, C$, etc. for a suitable positive constants unless indicated otherwise.

\section{Regularity}

In this section, we prove the regularity of solutions to system (4). To this end, we need the following regularity lifting lemma (see [Chen and Li 2010; Ma et al. 2011]). An earlier version was introduced in [Chen and Li 2005].

Let $V$ be a topological vector space. Suppose there are two extended norms (i.e., the norm of an element in $V$ might be infinity) defined on $V$,

$$
\|\cdot\|_{X},\|\cdot\|_{Y}: V \rightarrow[0, \infty]
$$


Let

$$
X:=\left\{f \in V:\|f\|_{X}<\infty\right\} \quad \text { and } \quad Y:=\left\{f \in V:\|f\|_{Y}<\infty\right\} .
$$

Lemma 2.1. Let $T$ be a contraction map from $X$ into itself and from $Y$ into itself. Assume that for any $f \in X$ there exists a function $g \in Z:=X \cap Y$ such that $f=T f+g$ in $X$. Then $f \in Z$.

We also need an equivalent form of the HLS inequality (see [Chen and Li 2005; 2010]): let $C(n, \alpha, p)$ be a uniform positive constant and define

$$
T f(x)=\int_{\mathbb{R}^{n}} \frac{f(y)}{|x-y|^{n-\alpha}} d y .
$$

Assume that $f \in L^{p}\left(\mathbb{R}^{n}\right)$ for $\frac{n}{n-\alpha}<p<\infty$. Then

$$
\|T f\|_{L^{p}\left(\mathbb{R}^{n}\right)} \leq C(n, \alpha, p)\|f\|_{L^{\frac{n p}{n+\alpha p}}\left(\mathbb{R}^{n}\right)} .
$$

Denote

$$
u_{R}(x)= \begin{cases}u(x), & |u(x)|>R \\ 0, & |u(x)| \leq R .\end{cases}
$$

Assume that $\phi \in L^{r}\left(\mathbb{R}^{n}\right), \varphi \in L^{s}\left(\mathbb{R}^{n}\right)$ for $\frac{n}{n-\alpha}<r, s<\infty$. Define

$$
\begin{aligned}
& T_{1}(\phi, \varphi)=\int_{\mathbb{R}^{n}} \frac{\lambda_{1} u_{R}^{p_{1}-1}(y)}{|x-y|^{n-\alpha}} \phi(y) d y+\int_{\mathbb{R}^{n}} \frac{\mu_{1} v_{R}^{p_{2}-1}(y)+\beta_{1} u_{R}^{p_{3}}(y) v_{R}^{p_{4}-1}(y)}{|x-y|^{n-\alpha}} \varphi(y) d y, \\
& T_{2}(\phi, \varphi)=\int_{\mathbb{R}^{n}} \frac{\mu_{2} v_{R}^{q_{2}-1}(y)}{|x-y|^{n-\alpha}} \varphi(y) d y+\int_{\mathbb{R}^{n}} \frac{\lambda_{2} u_{R}^{q_{1}-1}(y)+\beta_{2} v_{R}^{q_{4}}(y) u_{R}^{q_{3}-1}(y)}{|x-y|^{n-\alpha}} \phi(y) d y .
\end{aligned}
$$

Let $u_{b}(x)=u(x)-u_{R}(x)$, and

$$
\begin{aligned}
& f_{R}(x)=\int_{\mathbb{R}^{n}} \frac{\mu_{1} v_{b}^{p_{2}-1}(y)+\beta_{1} u_{b}^{p_{3}}(y) v_{b}^{p_{4}-1}(y)}{|x-y|^{n-\alpha}} v(y) d y+\int_{\mathbb{R}^{n}} \frac{\lambda_{1} u_{b}^{p_{1}-1}(y)}{|x-y|^{n-\alpha}} u(y) d y, \\
& g_{R}(x)=\int_{\mathbb{R}^{n}} \frac{\lambda_{2} u_{b}^{q_{1}-1}(y)+\beta_{2} v_{b}^{q_{4}}(y) u_{b}^{q_{3}-1}(y)}{|x-y|^{n-\alpha}} u(y) d y+\int_{\mathbb{R}^{n}} \frac{\mu_{2} v_{b}^{q_{2}-1}(y)}{|x-y|^{n-\alpha}} v(y) d y .
\end{aligned}
$$

Denote the norm in the cross product space $L^{r}\left(\mathbb{R}^{n}\right) \times L^{s}\left(\mathbb{R}^{n}\right)$ by

$$
\|(u, v)\|_{r \times s}=\|u\|_{r}+\|v\|_{s},
$$

and define the mapping $T: L^{r}\left(\mathbb{R}^{n}\right) \times L^{s}\left(\mathbb{R}^{n}\right) \rightarrow L^{r}\left(\mathbb{R}^{n}\right) \times L^{s}\left(\mathbb{R}^{n}\right)$ by

$$
T(\phi, \varphi)=\left(T_{1}(\phi, \varphi), T_{2}(\phi, \varphi)\right) .
$$

Throughout the paper, we use the notation $\|u\|_{s}=\|u\|_{L^{s}\left(\mathbb{R}^{n}\right)}$.

Consider the equation

$$
(\phi, \varphi)=T(\phi, \varphi)+\left(f_{R}, g_{R}\right)
$$


Notice that there is no intersection between the supports of $u_{R}, v_{R}$ and $u_{b}, v_{b}$, so $(u, v)$ is a pair of solutions of (13).

Proof of Theorem 1.1. The proof is divided into three steps.

Step 1. Firstly, we show $u, v \in L^{s}\left(\mathbb{R}^{n}\right)$ for all $s>\frac{n}{n-\alpha}$. To this end, we show that

(i) $T$ is a contracting map from $L^{s}\left(\mathbb{R}^{n}\right) \times L^{s}\left(\mathbb{R}^{n}\right)$ to itself for $R$ large enough;

(ii) $f_{R}$ and $g_{R}$ belong to $L^{s}\left(\mathbb{R}^{n}\right)$.

We first show (i). For any $\phi, \varphi \in L^{s}\left(\mathbb{R}^{n}\right)$, using the HLS inequality (12) and the Minkowski inequality, we have

(14) $\left\|T_{1}(\phi, \varphi)\right\|_{s}$

$$
\leq C(n, \alpha, \gamma)\left(\lambda_{1}\left\|u_{R}^{p_{1}-1} \phi\right\|_{\theta}+\mu_{1}\left\|v_{R}^{p_{2}-1} \varphi\right\|_{\theta}+\beta_{1}\left\|u_{R}^{p_{3}} v_{R}^{p_{4}-1} \varphi\right\|_{\theta}\right),
$$

where $\theta=\frac{n s}{n+\alpha s}$. By the Hölder inequality, we have

$$
\left\|u_{R}^{p_{1}-1} \phi\right\|_{\theta} \leq\left\|u_{R}\right\|_{s_{11}}^{p_{1}-1}\|\phi\|_{s}, \quad\left\|v_{R}^{p_{2}-1} \varphi\right\|_{\theta} \leq\left\|v_{R}\right\|_{s_{12}}^{p_{2}-1}\|\varphi\|_{s},
$$

where $s_{1 j}=n\left(p_{j}-1\right) / \alpha, j=1,2$, and

$$
\begin{aligned}
& \left\|u_{R}{ }^{p_{3}} v_{R}{ }^{p_{4}-1} \varphi\right\|_{\theta} \\
& \quad \leq\left(\int_{\mathbb{R}^{n}} u_{R}^{p_{3} t_{1} \theta}(y) d y\right)^{\frac{1}{t_{1} \theta}}\left(\int_{\mathbb{R}^{n}} v_{R}^{\left(p_{4}-1\right) t_{2} \theta}(y) d y\right)^{\frac{1}{t_{2} \theta}}\left(\int_{\mathbb{R}^{n}} \varphi^{t_{3} \theta}(y) d y\right)^{\frac{1}{t_{3} \theta}} \\
& \quad=\left\|u_{R}\right\|_{k_{0}}^{p_{3}}\left\|v_{R}\right\|_{k_{0}}^{p_{4}-1}\|\varphi\|_{s} .
\end{aligned}
$$

In the above inequality we have chosen $t_{3}=(n+s \alpha) / n>1$, so we take $1 / t_{1}+1 / t_{2}=$ $s \alpha /(n+s \alpha)$ with

$$
k_{0}=t_{1} p_{3} \theta=t_{2}\left(p_{4}-1\right) \theta,
$$

and then

$$
\frac{p_{3}}{k_{0}}+\frac{p_{4}-1}{k_{0}}=\frac{\alpha}{n} .
$$

Substituting (15) and (16) into (14), we deduce that

$$
\left\|T_{1}(\phi, \varphi)\right\|_{s} \leq c\left\|u_{R}\right\|_{s_{11}}^{p_{1}-1}\|\phi\|_{s}+c\left(\left\|v_{R}\right\|_{s_{12}}^{p_{2}-1}+\left\|u_{R}\right\|_{k_{0}}^{p_{3}}\left\|v_{R}\right\|_{k_{0}}^{p_{4}-1}\right)\|\varphi\|_{s} .
$$

Since $u \in L^{s_{11}}\left(\mathbb{R}^{n}\right) \cap L^{k_{0}}\left(\mathbb{R}^{n}\right), v \in L^{s_{12}}\left(\mathbb{R}^{n}\right) \cap L^{k_{0}}\left(\mathbb{R}^{n}\right)$, we may choose $R$ large enough such that

$$
\left\|u_{R}\right\|_{s_{11}}^{p_{2}-1} \leq \frac{1}{4}, \quad\left\|v_{R}\right\|_{s_{12}}^{p_{2}-1}+\left\|u_{R}\right\|_{k_{0}}^{p_{3}}\left\|v_{R}\right\|_{k_{0}}^{p_{4}-1} \leq \frac{1}{4} .
$$

Hence, from (17) we obtain

$$
\left\|T_{1}(\phi, \varphi)\right\|_{s} \leq \frac{1}{4}\left(\|\phi\|_{s}+\|\varphi\|_{s}\right) .
$$


Similarly, we have

$$
\left\|T_{2}(\phi, \varphi)\right\|_{s} \leq \frac{1}{4}\left(\|\phi\|_{s}+\|\varphi\|_{s}\right) .
$$

Combining (18) and (19), one obtains

$$
\|T(\phi, \varphi)\|_{s \times s} \leq \frac{1}{2}\left(\|\phi\|_{s}+\|\varphi\|_{s}\right) .
$$

It turns out that $T$ is the contracting map from $L^{s}\left(\mathbb{R}^{n}\right) \times L^{s}\left(\mathbb{R}^{n}\right)$ to itself.

(ii) Next we estimate $f_{R}$ and $g_{R}$. We write

$$
\begin{aligned}
f_{R}(x) & =\int_{\mathbb{R}^{n}} \frac{\mu_{1} v_{b}^{p_{2}-1}(y)+\beta_{1} u_{b}^{p_{3}}(y) v_{b}^{p_{4}-1}(y)}{|x-y|^{n-\alpha}} v(y) d y+\int_{\mathbb{R}^{n}} \frac{\lambda_{1} u_{b}^{p_{1}-1}(y)}{|x-y|^{n-\alpha}} u(y) d y \\
& =: J_{1}+J_{2} .
\end{aligned}
$$

For any $s>\frac{n}{n-\alpha}$, we apply the HLS inequality, Minkowski inequality and Hölder inequality to get

$$
\begin{aligned}
\left\|J_{1}\right\|_{s} & \leq c\left\|v_{b}{ }^{p_{2}-1} v\right\|_{\theta}+c\left\|u_{b}{ }^{p_{3}} v_{b}{ }^{p_{4}-1} v\right\|_{\theta} \\
& \leq c\left\|v_{b}\right\|_{k_{1}}^{p_{2}-1}\|v\|_{k_{2}}+c\left\|u_{b}\right\|_{k_{3}}^{p_{3}}\left\|v_{b}\right\|_{k_{4}}^{p_{4}-1}\|v\|_{k_{5}},
\end{aligned}
$$

and

$$
\left\|J_{2}\right\|_{s} \leq c\left\|u_{b}^{p_{1}-1} u\right\|_{\theta} \leq c\left\|u_{b}\right\|_{k_{6}}^{p_{1}-1}\|u\|_{k_{7}},
$$

where

$$
\frac{p_{2}-1}{k_{1}}+\frac{1}{k_{2}}=\frac{p_{3}}{k_{3}}+\frac{p_{4}-1}{k_{4}}+\frac{1}{k_{5}}=\frac{p_{1}-1}{k_{6}}+\frac{1}{k_{7}}=\frac{n+\alpha s}{n s}=\frac{1}{s}+\frac{\alpha}{n} .
$$

Since $v_{b}, u_{b}$ are bounded, $k_{1}, k_{3}, k_{4}, k_{6}$ can be chosen arbitrarily. Notice that $\frac{n}{n-\alpha}<$ $p_{3}+p_{4}=q_{3}+q_{4}$, so it follows that $k_{0}=n\left(p_{3}+p_{4}-1\right) / \alpha=n\left(q_{3}+q_{4}-1\right) / \alpha>$ $n /(n-\alpha)$. In view of $u, v \in L^{k_{0}}\left(\mathbb{R}^{n}\right)$, we may choose $k_{2}=k_{5}=k_{7}=k_{0}$ such that

$$
\begin{aligned}
\frac{1}{s} & =\frac{p_{2}-1}{k_{1}}+\frac{1}{k_{0}}-\frac{\alpha}{n}=\frac{p_{2}-1}{k_{1}}+\frac{n-\alpha k_{0}}{n k_{0}} \\
& =\frac{p_{3}}{k_{3}}+\frac{p_{4}-1}{k_{4}}+\frac{n-\alpha k_{0}}{n k_{0}} \\
& =\frac{p_{1}-1}{k_{6}}+\frac{n-\alpha k_{0}}{n k_{0}} .
\end{aligned}
$$

Now, letting $k_{1}, k_{3}, k_{4}, k_{6} \rightarrow \infty$, the previous equation implies that

$$
s \rightarrow \frac{n k_{0}}{n-\alpha k_{0}} .
$$

We conclude that $f_{R} \in L^{n k_{0} /\left(n-\alpha k_{0}\right)-\epsilon}\left(\mathbb{R}^{n}\right)$ for any small $\epsilon>0$. Obviously, $n k_{0} /$ $\left(n-\alpha k_{0}\right)>k_{0}$. Similarly, we can show $g_{R} \in L^{n k_{0} /\left(n-\alpha k_{0}\right)-\epsilon}\left(\mathbb{R}^{n}\right)$. 
By Lemma 2.1, if $n \leq \alpha k_{0}$, we are done. If $n>\alpha k_{0}$, we repeat the above process, and after a few steps, we obtain

$$
u, v \in L^{s}\left(\mathbb{R}^{n}\right), \quad \frac{n}{n-\alpha}<s<\infty .
$$

Step 2. We show $u, v \in L^{\infty}\left(\mathbb{R}^{n}\right)$. We split $u(x)$ into two parts, i.e., $u(x)$ can be written as

$$
\begin{aligned}
u(x)= & \int_{B_{1}(x)} \frac{\lambda_{1} u^{p_{1}}(y)+\mu_{1} v^{p_{2}}(y)+\beta_{1} u^{p_{3}}(y) v^{p_{4}}(y)}{|x-y|^{n-\alpha}} d y \\
& \quad+\int_{\mathbb{R}^{n} \backslash B_{1}(x)} \frac{\lambda_{1} u^{p_{1}}(y)+\mu_{1} v^{p_{2}}(y)+\beta_{1} u^{p_{3}}(y) v^{p_{4}}(y)}{|x-y|^{n-\alpha}} d y \\
= & : J_{3}+J_{4} .
\end{aligned}
$$

We estimate $J_{3}$ and $J_{4}$ separately. First consider $J_{4}$. Since $1 /|x-y|^{n-\alpha}<1$, and $u, v \in L^{s}\left(\mathbb{R}^{n}\right)$ for any $\frac{n}{n-\alpha}<s$, according to the assumptions that $\frac{n}{n-\alpha}<$ $p_{1}, p_{2}, p_{3}+p_{4}$, and using the Hölder inequality, we have

$$
\begin{aligned}
J_{4} & \leq c \int_{\mathbb{R}^{n} \backslash B_{1}(x)} u^{p_{1}}(y) d y+c \int_{\mathbb{R}^{n} \backslash B_{1}(x)} v^{p_{2}}(y) d y+c \int_{\mathbb{R}^{n} \backslash B_{1}(x)} u^{p_{3}}(y) v^{p_{4}}(y) d y \\
& <\infty .
\end{aligned}
$$

Next, we compute $J_{3}$ :

$$
\begin{aligned}
J_{3} \leq & c\left(\int_{B_{1}(x)} \frac{1}{|x-y|^{(n-\alpha) p}} d y\right)^{1 / p}\left(\int_{B_{1}(x)}|u(y)|^{p_{1} p /(p-1)} d y\right)^{(p-1) / p} \\
& +c\left(\int_{B_{1}(x)} \frac{1}{|x-y|^{(n-\alpha) p}} d y\right)^{1 / p}\left(\int_{B_{1}(x)}|v(y)|^{p_{2} p /(p-1)} d y\right)^{(p-1) / p} \\
& +c\left(\int_{B_{1}(x)} \frac{1}{|x-y|^{(n-\alpha) p}} d y\right)^{1 / p}\left(\int_{B_{1}(x)}\left(\left|u^{p_{3}}(y) v^{p_{4}}(y)\right|\right)^{p /(p-1)} d y\right)^{(p-1) / p} .
\end{aligned}
$$

Choose the constant $p$ such that $(n-\alpha) p<n$, and then

$$
\left(\int_{B_{1}(x)} \frac{1}{|x-y|^{(n-\alpha) p}} d y\right)^{1 / p}<C .
$$

Since $u, v \in L^{s}\left(\mathbb{R}^{n}\right)$ for any $\frac{n}{n-\alpha}<s<\infty$, we get

$$
\left(\int_{B_{1}(x)}|u(y)|^{p_{1} p /(p-1)} d y\right)^{(p-1) / p}<C, \quad\left(\int_{B_{1}(x)}|v(y)|^{p_{2} p /(p-1)} d y\right)^{(p-1) / p}<C,
$$


and by the Hölder inequality, we obtain

$$
\begin{aligned}
& \left(\int_{B_{1}(x)}\left|u^{p_{3}}(y) v^{p_{4}}(y)\right|^{\frac{p}{p-1}} d y\right)^{\frac{p-1}{p}} \\
& \leq\left(\int_{B_{1}(x)}|u(y)|^{p_{3} l_{1} \frac{p}{p-1}} d y\right)^{\frac{p-1}{l_{1} p}}\left(\int_{B_{1}(x)}|v(y)|^{p_{4} l_{2} \frac{p}{p-1}} d y\right)^{\frac{p-1}{l_{2} p}}<C,
\end{aligned}
$$

where $l_{1}, l_{2}>1$ and $1 / l_{1}+1 / l_{2}=1$. (We may choose $l_{1}=\left(p_{3}+p_{4}\right) / p_{3}$ and $l_{2}=\left(p_{3}+p_{4}\right) / p_{4}$.) So $u \in L^{\infty}\left(\mathbb{R}^{n}\right)$. Arguing as above, it also follows that $v \in L^{\infty}\left(\mathbb{R}^{n}\right)$.

Step 3. Using the usual bootstrap method, as in [Li 2004], we conclude $u, v \in$ $C^{\infty}\left(\mathbb{R}^{n}\right)$.

\section{Radial symmetry and monotonicity}

In this section, we use the method of moving plane in integral form to prove Theorem 1.2. The moving plane method in integral form used here was introduced by $\mathrm{Chen}, \mathrm{Li}$ and $\mathrm{Ou}$ [2006] and exploits global properties of integral equations instead of using the amount of local properties of differential operators as the traditional moving plane method (e.g., see [Guo and Liu 2008; de Figueiredo and Sirakov 2005; Liu et al. 2006; Zhang 2007; Wei and Xu 1999; Gidas et al. 1981]).

We first deduce two representation formulas related to $u(x)$ and $v(x)$, respectively. Let $\lambda$ be a real number. Define

$$
\Sigma_{\lambda}=\left\{x=\left(x_{1} \cdots x_{n}\right) \mid x_{1} \geq \lambda\right\},
$$

and set

$$
x^{\lambda}=\left(2 \lambda-x_{1}, x_{2}, \ldots, x_{n}\right), \quad u_{\lambda}(x)=u\left(x^{\lambda}\right) \quad \text { and } \quad v_{\lambda}(x)=v\left(x^{\lambda}\right) .
$$

For convenience, we set $Q_{y}(u, v)=\lambda_{1} u^{p_{1}}(y)+\mu_{1} v^{p_{2}}(y)+\beta_{1} u^{p_{3}}(y) v^{p_{4}}(y)$ and $K_{y}(u, v)=\lambda_{2} u^{q_{1}}(y)+\mu_{2} v^{q_{2}}(y)+\beta_{2} u^{q_{3}}(y) v^{q_{4}}(y)$. In view of (4), we have

$$
\begin{aligned}
u_{\lambda}(x) & =\int_{\mathbb{R}^{n}} \frac{Q_{y}(u, v)}{\left|x^{\lambda}-y\right|^{n-\alpha}} d y \\
& =\int_{\Sigma_{\lambda}} \frac{Q_{y}(u, v)}{\left|x^{\lambda}-y\right|^{n-\alpha}} d y+\int_{\mathbb{R}^{n} \backslash \Sigma_{\lambda}} \frac{Q_{y}(u, v)}{\left|x^{\lambda}-y\right|^{n-\alpha}} d y \\
& =\int_{\Sigma_{\lambda}} \frac{Q_{y}(u, v)}{\left|x^{\lambda}-y\right|^{n-\alpha}} d y+\int_{\Sigma_{\lambda}} \frac{Q_{y}\left(u_{\lambda}, v_{\lambda}\right)}{\left|x^{\lambda}-y^{\lambda}\right|^{n-\alpha}} d y .
\end{aligned}
$$

We also have

$$
v_{\lambda}(x)=\int_{\Sigma_{\lambda}} \frac{K_{y}(u, v)}{\left|x^{\lambda}-y\right|^{n-\alpha}} d y+\int_{\Sigma_{\lambda}} \frac{K_{y}\left(u_{\lambda}, v_{\lambda}\right)}{\left|x^{\lambda}-y^{\lambda}\right|^{n-\alpha}} d y .
$$


Noting that $\left|x^{\lambda}-y^{\lambda}\right|=|x-y|$, it is easy to see that

$$
\begin{aligned}
& u_{\lambda}(x)-u(x) \\
& =\int_{\Sigma_{\lambda}}\left(\frac{1}{|x-y|^{n-\alpha}}-\frac{1}{\left|x^{\lambda}-y\right|^{n-\alpha}}\right)\left(Q_{y}\left(u_{\lambda}, v_{\lambda}\right)-Q_{y}(u, v)\right) d y \\
& =\int_{\Sigma_{\lambda}}\left(\frac{1}{|x-y|^{n-\alpha}}-\frac{1}{\left|x^{\lambda}-y\right|^{n-\alpha}}\right)\left[\begin{array}{c}
\lambda_{1}\left(u_{\lambda}^{p_{1}}(y)-u^{p_{1}}(y)\right)+\mu_{1}\left(v_{\lambda}^{p_{2}}(y)-v^{p_{2}}(y)\right) \\
\left.+\beta_{1}\left(u_{\lambda}^{p_{3}}(y) v_{\lambda}^{p_{4}}(y)-u^{p_{3}}(y) v^{p_{4}}(y)\right)\right] d y
\end{array}\right.
\end{aligned}
$$

and

(24)

$$
\begin{aligned}
& v_{\lambda}(x)-v(x) \\
& =\int_{\Sigma_{\lambda}}\left(\frac{1}{|x-y|^{n-\alpha}}-\frac{1}{\left|x^{\lambda}-y\right|^{n-\alpha}}\right)\left(K_{y}\left(u_{\lambda}, v_{\lambda}\right)-K_{y}(u, v)\right) d y \\
& =\int_{\Sigma_{\lambda}}\left(\frac{1}{|x-y|^{n-\alpha}}-\frac{1}{\left|x^{\lambda}-y\right|^{n-\alpha}}\right)\left[\begin{array}{c}
\lambda_{2}\left(u_{\lambda}^{q_{1}}(y)-u^{q_{1}}(y)\right)+\mu_{2}\left(v_{\lambda}^{q_{2}}(y)-v^{q_{2}}(y)\right) \\
\left.+\beta_{2}\left(u_{\lambda}^{q_{3}}(y) v_{\lambda}^{q_{4}}(y)-u^{q_{3}}(y) v^{q_{4}}(y)\right)\right] d y .
\end{array}\right.
\end{aligned}
$$

The next lemma shows the plane can start moving from $x_{1}=-\infty$ to the right.

Lemma 3.1. Let $(u, v) \in \Pi_{1} \times \Pi_{2}$ be a pair of positive solutions of (4). Then, for $\lambda$ sufficiently negative,

$$
u(x) \geq u_{\lambda}(x) \quad \text { and } \quad v(x) \geq v_{\lambda}(x) \text { for all } x \in \Sigma_{\lambda} .
$$

Proof. Define

$$
\Sigma_{\lambda}^{u}=\left\{x \in \Sigma_{\lambda} \mid u(x)<u_{\lambda}(x)\right\} \quad \text { and } \quad \Sigma_{\lambda}^{v}=\left\{x \in \Sigma_{\lambda} \mid v(x)<v_{\lambda}(x)\right\} .
$$

Let $\Sigma_{\lambda}^{c}$ be the complement of $\Sigma_{\lambda}$. From (23) and the mean value theorem, we have (26)

$$
\begin{aligned}
& u_{\lambda}(x)-u(x) \\
& =\int_{\Sigma_{\lambda}}\left(\frac{1}{|x-y|^{n-\alpha}}-\frac{1}{\left|x^{\lambda}-y\right|^{n-\alpha}}\right) \\
& \quad \times\left[\lambda_{1}\left(u_{\lambda}^{p_{1}}(y)-u^{p_{1}}(y)\right)+\mu_{1}\left(v_{\lambda}^{p_{2}}(y)-v^{p_{2}}(y)\right)\right. \\
& \left.\quad+\beta_{1}\left(u_{\lambda}^{p_{3}}(y)\left(v_{\lambda}^{p_{4}}(y)-v^{p_{4}}(y)\right)+v^{p_{4}}(y)\left(u_{\lambda}^{p_{3}}(y)-u^{p_{3}}(y)\right)\right)\right] d y \\
& \leq \int_{\Sigma_{\lambda}}\left(\frac{1}{|x-y|^{n-\alpha}}-\frac{1}{\left|x^{\lambda}-y\right|^{n-\alpha}}\right) \\
& \quad \times\left[p_{1} \lambda_{1} \phi_{1}^{p_{1}-1}(u)\left(u_{\lambda}(y)-u(y)\right)+p_{2} \mu_{1} \phi_{2}^{p_{2}-1}(v)\left(v_{\lambda}(y)-v(y)\right)\right. \\
& +p_{4} \beta_{1} u_{\lambda}^{p_{3}}(y) \phi_{4}^{p_{4}-1}(v)\left(v_{\lambda}(y)-v(y)\right) \\
& \left.+p_{3} \beta_{1} v^{p_{4}}(y) \phi_{3}^{p_{3}-1}(u)\left(u_{\lambda}(y)-u(y)\right)\right] d y,
\end{aligned}
$$


where $u(y) \leq \phi_{i}(u) \leq u_{\lambda}(y), i=1,3$, on $\Sigma_{\lambda}^{u}$, and $v(y) \leq \phi_{j}(v) \leq v_{\lambda}(y), j=2,4$ on $\Sigma_{\lambda}^{v}$. It follows that we can write

$$
u_{\lambda}(x)-u(x) \leq c\left(I_{1}+I_{2}+I_{3}+I_{4}\right),
$$

where

$$
\begin{aligned}
I_{1} & :=\int_{\Sigma_{\lambda}^{u}} \frac{u_{\lambda}^{p_{1}-1}(y)}{|x-y|^{n-\alpha}}\left(u_{\lambda}(y)-u(y)\right) d y, \quad I_{2}:=\int_{\Sigma_{\lambda}^{v}} \frac{v_{\lambda}^{p_{2}-1}(y)}{|x-y|^{n-\alpha}}\left(v_{\lambda}(y)-v(y)\right) d y, \\
I_{3} & :=\int_{\Sigma_{\lambda}^{v}} \frac{u_{\lambda}^{p_{3}}(y) v_{\lambda}^{p_{4}-1}(y)}{|x-y|^{n-\alpha}}\left(v_{\lambda}(y)-v(y)\right) d y, \\
I_{4} & :=\int_{\Sigma_{\lambda}^{u}} \frac{v^{p_{4}}(y) u_{\lambda}^{p_{3}-1}(y)}{|x-y|^{n-\alpha}}\left(u_{\lambda}(y)-u(y)\right) d y .
\end{aligned}
$$

Using the HLS inequality and the Hölder inequality, we get

$$
\left(\int_{\Sigma_{\lambda}^{u}}\left|I_{1}\right|^{\gamma}\right)^{1 / \gamma} \leq C(n, \alpha, \gamma)\left\|u_{\lambda}^{p_{1}-1}\left(u_{\lambda}-u\right)\right\|_{L^{\theta}\left(\Sigma_{\lambda}^{u}\right)}
$$

for any $\frac{n}{n-\alpha}<\gamma<\infty$ and $\theta=\frac{n \gamma}{n+\alpha \gamma}$. Let $m_{1}=\frac{n+\alpha \gamma}{\alpha \gamma}>1$ and $m_{2}=\frac{n+\alpha \gamma}{n}>1$. Thus, we invoke the Hölder inequality to obtain

$$
\begin{aligned}
& \left\{\int_{\Sigma_{\lambda}^{u}}\left[u_{\lambda} p_{1}-1(y)\left(u_{\lambda}(y)-u(y)\right)\right]^{\theta} d y\right\}^{1 / \theta} \\
& \quad \leq\left\{\left[\int_{\Sigma_{\lambda}^{u}}\left(u_{\lambda}(y)\right)^{\theta\left(p_{1}-1\right) m_{1}} d y\right]^{1 / m_{1}}\left[\int_{\Sigma_{\lambda}^{u}}\left(u_{\lambda}(y)-u(y)\right)^{\theta m_{2}} d y\right]^{1 / m_{2}}\right\}^{1 / \theta} \\
& \quad=\left\|u_{\lambda}\right\|_{L_{\left(\Sigma_{\lambda}^{u}\right)}^{s_{11}-1}\left\|u_{\lambda}-u\right\|_{L_{\left(\Sigma_{\lambda}^{u}\right)}^{\gamma}},}^{p_{1}^{u}}
\end{aligned}
$$

where $s_{11}=n\left(p_{1}-1\right) / \alpha$. Substituting (28) into (27), we get

$$
\left(\int_{\Sigma_{\lambda}^{u}}\left|I_{1}\right|^{\gamma}\right)^{1 / \gamma} \leq C(n, \alpha, \gamma)\left\|u_{\lambda}\right\|_{L_{\left(\Sigma_{\lambda}^{u}\right)}^{s_{11}}}^{p_{1}-1}\left\|u_{\lambda}-u\right\|_{L_{\left(\Sigma_{\lambda}^{u}\right)}^{\gamma}} .
$$

Similarly, one has

$$
\left\|I_{2}\right\|_{L_{\left(\Sigma_{\lambda}^{u}\right)}^{\gamma}} \leq C(n, \alpha, \gamma)\left\|v_{\lambda}\right\|_{L_{\left(\Sigma_{\lambda}^{v}\right)}^{s_{12}}}^{p_{2}-1}\left\|v_{\lambda}-v\right\|_{L_{\left(\Sigma_{\lambda}^{v}\right)}^{\gamma}},
$$

where $s_{12}=n\left(p_{2}-1\right) / \alpha$.

Next, we estimate $I_{3}$ and $I_{4}$. By the HLS inequality we have

$$
\left\|I_{3}\right\|_{L_{\left(\Sigma_{\lambda}^{u}\right)}^{\gamma}} \leq C(n, \alpha, \gamma)\left\|u_{\lambda}^{p_{3}} v_{\lambda}^{p^{4}-1}\left(v_{\lambda}-v\right)\right\|_{L_{\left(\Sigma_{\lambda}^{v}\right)}^{\theta}} .
$$

Letting $1 / t_{1}+1 / t_{2}+1 / t_{3}=1$ for $t_{1}>1$, it follows that 
(32)

$$
\begin{aligned}
& \left\|u_{\lambda}^{p_{3}} v_{\lambda}^{p_{4}-1}\left(v_{\lambda}-v\right)\right\|_{L_{\left(\Sigma_{\lambda}^{v}\right)}^{\theta}} \\
& \quad \leq\left[\int_{\Sigma_{\lambda}^{v}} u_{\lambda}^{p_{3} \theta t_{1}}(y) d y\right]^{\frac{1}{t_{1} \theta}}\left[\int_{\Sigma_{\lambda}^{v}} v_{\lambda}^{\left(p_{4}-1\right) \theta t_{2}}(y) d y\right]^{\frac{1}{t_{2} \theta}}\left[\int_{\Sigma_{\lambda}^{v}}\left(v_{\lambda}(y)-v(y)\right)^{\theta t_{3}} d y\right]^{\frac{1}{t_{3} \theta}} \\
& \leq\left\|u_{\lambda}\right\|_{L_{\left(\Sigma_{\lambda}^{v}\right)}^{k_{3}}}^{p_{3}}\left\|v_{\lambda}\right\|_{L_{\left(\Sigma_{\lambda}^{v}\right)}^{k_{0}-1}\left\|v_{\lambda}-v\right\|_{L_{\left(\Sigma_{\lambda}^{v}\right)}^{v}}^{p^{v}}}
\end{aligned}
$$

Arguing as Section 2, we choose $t_{3}=(n+\alpha \gamma) / n>1, t_{1}=(n+\alpha \gamma)\left(p_{3}+p_{4}-1\right) /$ $\left(p_{3} \alpha \gamma\right)$ and $t_{2}=(n+\alpha \gamma)\left(p_{3}+p_{4}-1\right) /\left(\left(p_{4}-1\right) \alpha \gamma\right)$, satisfying $1 / t_{1}+1 / t_{2}+1 / t_{3}=1$. Then $k_{0}=t_{1} p_{3} \theta=t_{2}\left(p_{4}-1\right) \theta$. Substituting (32) into (31), we conclude

$$
\left\|I_{3}\right\|_{L_{\left(\Sigma_{\lambda}^{u}\right)}^{\gamma}}^{\gamma} \leq C(n, \alpha, \gamma)\left\|u_{\lambda}\right\|_{L_{\left(\Sigma_{\lambda}^{v}\right)}^{k_{0}}}^{p_{3}}\left\|v_{\lambda}\right\|_{L_{\left(\Sigma_{\lambda}^{v}\right)}^{k_{0}}}^{p_{0}-1}\left\|v_{\lambda}-v\right\|_{L_{\left(\Sigma_{\lambda}^{v}\right)}^{\gamma}}
$$

In the same way, one has

$$
\left\|I_{4}\right\|_{L_{\left(\Sigma_{\lambda}^{u}\right)}^{\gamma}} \leq C(n, \alpha, \gamma)\|v\|_{L_{\left(\Sigma_{\lambda}^{k_{0}}\right)}^{k_{0}}}^{p_{4}}\left\|u_{\lambda}\right\|_{L_{\left(\Sigma_{\lambda}^{u}\right)}^{k_{0}}}^{p_{3}-1}\left\|u_{\lambda}-u\right\|_{L_{\left(\Sigma_{\lambda}^{u}\right)}^{\gamma}}
$$

Now, we compute the norm $L^{\gamma}\left(\Sigma_{\lambda}^{u}\right)$ of (26) for any $\frac{n}{n-\alpha}<\gamma<\infty$. Using the Minkowski inequality and combining (29), (30), (33) and (34), we arrive at

$$
\begin{aligned}
& \left\|u_{\lambda}-u\right\|_{L_{\left(\Sigma_{\lambda}^{u}\right)}^{\gamma}} \\
& \leq c\left\|u_{\lambda}\right\|_{L_{\left(\Sigma_{\lambda}^{u}\right)}^{s_{11}^{u}}}^{p_{1}-1}\left\|u_{\lambda}-u\right\|_{L_{\left(\Sigma_{\lambda}^{u}\right)}^{\gamma}}+c\left\|v_{\lambda}\right\|_{L_{\left(\Sigma_{\lambda}^{v}\right)}^{s_{12}}}^{p_{2}-1}\left\|v_{\lambda}-v\right\|_{L_{\left(\Sigma_{\lambda}^{v}\right)}^{\gamma}} \\
& +c\left\|u_{\lambda}\right\|_{L_{\left(\Sigma_{\lambda}^{v}\right)}^{k_{0}}}^{p_{3}}\left\|v_{\lambda}\right\|_{L_{\left(\Sigma_{\lambda}^{v}\right)}^{k_{0}}}^{p_{4}-1}\left\|v_{\lambda}-v\right\|_{L_{\left(\Sigma_{\lambda}^{v}\right)}^{\gamma}}+c\|v\|_{L_{\left(\Sigma_{\lambda}^{u}\right)}^{k_{0}}}^{p_{4}}\left\|u_{\lambda}\right\|_{L_{\left(\Sigma_{\lambda}^{u}\right)}^{k_{0}}}^{p_{3}-1}\left\|u_{\lambda}-u\right\|_{L_{\left(\Sigma_{\lambda}^{u}\right)}^{\gamma}}
\end{aligned}
$$

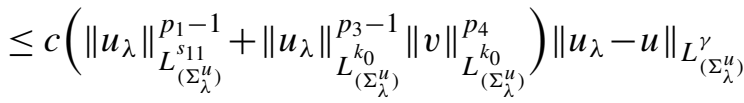

$$
\begin{aligned}
& +c\left(\left\|v_{\lambda}\right\|_{L_{\left(\Sigma_{\lambda}^{v}\right)}^{s_{12}^{v}}}^{p_{2}-1}+\left\|u_{\lambda}\right\|_{L_{\left(\Sigma_{\lambda}^{v}\right)}^{k_{0}^{v}}}^{p_{3}}\left\|v_{\lambda}\right\|_{L_{\left(\Sigma_{\lambda}^{v}\right)}^{k_{0}}}^{p_{4}-1}\right)\left\|v_{\lambda}-v\right\|_{L_{\left(\Sigma_{\lambda}^{v}\right)}^{\gamma}} .
\end{aligned}
$$

Along the same line, noting that $p_{3}+p_{4}=q_{3}+q_{4}$, we have

$$
\begin{aligned}
& \left\|v_{\lambda}-v\right\|_{L_{\left(\Sigma_{\lambda}^{v}\right)}^{\gamma}} \leq c\left(\left\|u_{\lambda}\right\|_{L_{\left(\Sigma_{\lambda}^{u}\right)}^{s_{2}}}^{q_{1}-1}+\left\|u_{\lambda}\right\|_{L_{\left(\Sigma_{\lambda}^{u}\right)}^{k_{0}}}^{q_{3}-1}\|v\|_{L_{\left(\Sigma_{\lambda}^{u}\right)}^{k_{0}}}^{q_{4}}\right)\left\|u_{\lambda}-u\right\|_{L_{\left(\Sigma_{\lambda}^{u}\right)}^{\gamma}} \\
& +c\left(\left\|v_{\lambda}\right\|_{L_{\left(\Sigma_{\lambda}^{v}\right)}^{s_{22}}}^{q_{2}-1}+\left\|u_{\lambda}\right\|_{L_{\left(\Sigma_{\lambda}^{v}\right)}^{k_{0}}}^{q_{3}}\left\|v_{\lambda}\right\|_{L_{\left(\Sigma_{\lambda}^{v}\right)}^{k_{0}}}^{q_{4}-1}\right)\left\|v_{\lambda}-v\right\|_{L_{\left(\Sigma_{\lambda}^{v}\right)}^{\gamma}},
\end{aligned}
$$

where $s_{21}=n\left(q_{1}-1\right) / \alpha, s_{22}=n\left(q_{2}-1\right) / \alpha, k_{0}=n\left(q_{3}+q_{4}-1\right) / \alpha$. 
By adding (35) and (36), we obtain

$$
\begin{aligned}
& \left\|u_{\lambda}-u\right\|_{L_{\left(\Sigma_{\lambda}^{u}\right)}^{\gamma}}+\left\|v_{\lambda}-v\right\|_{L_{\left(\Sigma_{\lambda}^{v}\right)}^{\gamma}} \\
& \leq c\left(\left\|u_{\lambda}\right\|_{L_{\left(\Sigma_{\lambda}^{u}\right)}^{s_{11}}}^{p_{1}-1}+\left\|u_{\lambda}\right\|_{L_{\left(\Sigma_{\lambda}^{u}\right)}^{s_{21}}}^{q_{1}-1}+\|v\|_{L_{\left(\Sigma_{\lambda}^{u}\right)}^{k_{0}}}^{p_{4}}\left\|u_{\lambda}\right\|_{L_{\left(\Sigma_{\lambda}^{u}\right)}^{k_{0}}}^{p_{3}-1}+\|v\|_{L_{\left(\Sigma_{\lambda}^{u}\right)}^{k_{0}}}^{q_{4}}\left\|u_{\lambda}\right\|_{L_{\left(\Sigma_{\lambda}^{u}\right)}^{q_{0}}}^{q_{3}-1}\right) \\
& \times\left\|u_{\lambda}-u\right\|_{L_{\left(\Sigma_{\lambda}^{u}\right)}^{\nu}} \\
& +c\left(\left\|v_{\lambda}\right\|_{L_{\left(\Sigma_{\lambda}^{v}\right)}^{s_{12}}}^{p_{2}-1}+\left\|v_{\lambda}\right\|_{L_{\left(\Sigma_{\lambda}^{v}\right)}^{s_{2}}}^{q_{2}-1}+\left\|u_{\lambda}\right\|_{L_{\left(\Sigma_{\lambda}^{v}\right)}^{k_{0}}}^{p_{3}}\left\|v_{\lambda}\right\|_{L_{\left(\Sigma_{\lambda}^{v}\right)}^{k_{0}}}^{p_{4}-1}+\left\|u_{\lambda}\right\|_{L_{\left(\Sigma_{\lambda}^{v}\right)}^{k_{0}}}^{q_{3}}\left\|v_{\lambda}\right\|_{L_{\left(\Sigma_{\lambda}^{v}\right)}^{k_{0}}}^{q_{4}-1}\right) \\
& \times\left\|v_{\lambda}-v\right\|_{L_{\left(\Sigma_{\lambda}^{v}\right)}^{\gamma}} \\
& \leq c\left(\|u\|_{L_{\left(\Sigma_{\lambda}^{c}\right)}^{s_{11}}}^{p_{1}-1}+\|u\|_{L_{\left(\Sigma_{\lambda}^{c}\right)}^{s_{21}}}^{q_{1}-1}+\|v\|_{L_{\left(\Sigma_{\lambda}^{u}\right)}^{k_{0}}}^{p_{4}}\|u\|_{L_{\left(\Sigma_{\lambda}^{c}\right)}^{p_{0}-1}}^{p_{3}-1}+\|v\|_{L_{\left(\Sigma_{\lambda}^{u}\right)}^{k_{0}}}^{q_{4}}\|u\|_{L_{\left(\Sigma_{\lambda}^{c}\right)}^{k_{0}}}^{q_{3}-1}\right) \\
& \times\left\|u_{\lambda}-u\right\|_{L_{\left(\Sigma_{\lambda}^{u}\right)}^{\nu}} \\
& +c\left(\|v\|_{L_{\left(\Sigma_{\lambda}^{c}\right)}^{s_{12}}}^{p_{2}-1}+\|v\|_{L_{\left(\Sigma_{\lambda}^{c}\right)}^{s_{22}}}^{q_{2}-1}+\|u\|_{L_{\left(\Sigma_{\lambda}^{c}\right)}^{k_{0}}}^{p_{3}}\|v\|_{L_{\left(\Sigma_{\lambda}^{c}\right)}^{k_{0}}}^{p_{1}-1}+\|u\|_{L_{\left(\Sigma_{\lambda}^{c}\right)}^{k_{0}}}^{q_{3}}\|v\|_{L_{\left(\Sigma_{\lambda}^{c}\right)}^{k_{0}}}^{q_{4}-1}\right) \\
& \times\left\|v_{\lambda}-v\right\|_{L_{\left(\Sigma_{\lambda}^{v}\right)}^{\gamma}} .
\end{aligned}
$$

Since $u \in \Pi_{1}$ and $v \in \Pi_{2}$, we can choose $\lambda$ sufficiently negative such that

$$
\begin{aligned}
& c\left(\|u\|_{L_{\left(\Sigma_{\lambda}^{c}\right)}^{s_{11}}}^{p_{1}-1}+\|u\|_{L_{\left(\Sigma_{\lambda}^{c}\right)}^{s_{21}}}^{q_{1}-1}+\|v\|_{L_{\left(\Sigma_{\lambda}^{u}\right)}^{k_{0}}}^{p_{4}}\|u\|_{L_{\left(\Sigma_{\lambda}^{c}\right)}^{k_{0}^{c}}}^{p_{3}-1}+\|v\|_{L_{\left(\Sigma_{\lambda}^{u}\right)}^{k_{0}}}^{q_{4}}\|u\|_{L_{\left(\Sigma_{\lambda}^{c}\right)}^{k_{0}^{c}}}^{q_{3}-1}\right) \leq \frac{1}{2}, \\
& c\left(\|v\|_{L_{\left(\Sigma_{\lambda}^{c}\right)}^{s_{12}}}^{p_{2}-1}+\|v\|_{L_{\left(\Sigma_{\lambda}^{c}\right)}^{s_{2}}}^{q_{2}-1}+\|u\|_{L_{\left(\Sigma_{\lambda}^{c}\right)}^{k_{0}}}^{p_{3}}\|v\|_{L_{\left(\Sigma_{\lambda}^{c}\right)}^{k_{0}}}^{p_{4}-1}+\|u\|_{L_{\left(\Sigma_{\lambda}^{c}\right)}^{k_{0}}}^{q_{3}}\|v\|_{L_{\left(\Sigma_{\lambda}^{c}\right)}^{q_{1}-1}}^{q_{1}}\right) \leq \frac{1}{2} .
\end{aligned}
$$

Hence

$$
\left\|u_{\lambda}-u\right\|_{L_{\left(\Sigma_{\lambda}^{u}\right)}^{\gamma}}+\left\|v_{\lambda}-v\right\|_{L_{\left(\Sigma_{\lambda}^{v}\right)}^{\gamma}} \leq \frac{1}{2}\left\|u_{\lambda}-u\right\|_{L_{\left(\Sigma_{\lambda}^{u}\right)}^{\gamma}}+\frac{1}{2}\left\|v_{\lambda}-v\right\|_{L_{\left(\Sigma_{\lambda}^{v}\right)}^{\gamma}} .
$$

This implies that $\left\|u_{\lambda}-u\right\|_{L_{\left(\Sigma_{\lambda}^{u}\right)}^{\gamma}}=\left\|v_{\lambda}-v\right\|_{L_{\left(\Sigma_{\lambda}^{v}\right)}^{\gamma}}=0$, and therefore $\Sigma_{\lambda}^{u}$ and $\Sigma_{\lambda}^{v}$ must be empty. Thus, (25) is proved.

Next we define

$$
\lambda_{0}=\sup \left\{\lambda \in \mathbb{R} \mid u_{\mu}(x) \leq u(x), v_{\mu}(x) \leq v(x) \text { for all } \mu \leq \lambda \text { and all } x \in \Sigma_{\mu}\right\} .
$$

By the regularity of positive solutions to system (4), we observe the fact that $u$ and $v$ are bounded as $|x| \rightarrow \infty$. Combining this and noting $u, v>0$, we conclude $\lambda_{0}<\infty$. Thus, we will move the plane to the limiting position to derive symmetry. That is, we have the following lemma.

Lemma 3.2. Under the assumptions of Theorem 1.2, we have

$$
u_{\lambda_{0}}(x) \equiv u(x) \quad \text { and } \quad v_{\lambda_{0}}(x) \equiv v(x) \quad \text { for all } x \in \Sigma_{\lambda_{0}}
$$


Proof. We use argument by contradiction. Assume that there exists a $\lambda_{0}<0$ such that $u(x) \geq u_{\lambda_{0}}(x)$, and $v_{\lambda}(x) \geq v_{\lambda_{0}}(x)$, but $u(x) \not \equiv u_{\lambda_{0}}(x)$ or $v_{\lambda}(x) \not \equiv v_{\lambda_{0}}(x)$ for any $x \in \Sigma_{\lambda_{0}}$.

We show that the plane can be moved further to the right. More precisely, there exists an $\varepsilon$ depending on $n, \alpha$, and the solution $(u, v)$ itself such that

$$
u(x) \geq u_{\lambda}(x) \quad \text { and } \quad v(x) \geq v_{\lambda}(x), \quad \text { on } \Sigma_{\lambda}
$$

for $\lambda \in\left[\lambda_{0}, \lambda_{0}+\varepsilon\right)$.

In the case of $v(x) \not \equiv v_{\lambda_{0}}(x)$ on $\Sigma_{\lambda_{0}}$, from (23) and (24) we obtain $u(x) \not \equiv u_{\lambda_{0}}(x)$, that is, $u(x)>u_{\lambda_{0}}(x)$ in the interior of $\Sigma_{\lambda_{0}}$. Let

$$
\overline{\Sigma_{\lambda_{0}}^{u}}=\left\{x \in \Sigma_{\lambda_{0}} \mid u(x) \leq u_{\lambda_{0}}(x)\right\} \quad \text { and } \quad \overline{\Sigma_{\lambda_{0}}^{v}}=\left\{x \in \Sigma_{\lambda_{0}} \mid v(x) \leq v_{\lambda_{0}}(x)\right\} .
$$

Obviously, $\overline{\Sigma_{\lambda_{0}}^{u}}$ has measure zero, and $\lim _{\lambda \rightarrow \lambda_{0}} \Sigma_{\lambda}^{u} \subset \overline{\Sigma_{\lambda_{0}}^{u}}$. The same fact holds for that of $v$. Let $\left(\Sigma_{\lambda}^{u}\right)^{*}$ be the reflection of set $\Sigma_{\lambda}^{u}$ about the plane $x_{1}=\lambda$. Similarly to (37), we have

$$
\begin{aligned}
& \left\|u_{\lambda}-u\right\|_{L_{\left(\Sigma_{\lambda}^{u}\right)}^{\gamma}}+\left\|v_{\lambda}-v\right\|_{L_{\left(\Sigma_{\lambda}^{v}\right)}^{\gamma}} \\
& \leq c\left(\left\|u_{\lambda}\right\|_{L_{\left(\Sigma_{\lambda}^{u}\right)}^{s_{11}}}^{p_{1}-1}+\left\|u_{\lambda}\right\|_{L_{\left(\Sigma_{\lambda}^{u}\right)}^{s_{21}}}^{q_{1}-1}+\|v\|_{L_{\left(\Sigma_{\lambda}^{u}\right)}^{k_{2}}}^{p_{4}}\left\|u_{\lambda}\right\|_{L_{\left(\Sigma_{\lambda}^{u}\right)}^{p_{3}-1}}^{p_{0}-1}+\|v\|_{L_{\left(\Sigma_{\lambda}^{u}\right)}^{k_{0}}}^{q_{4}}\left\|u_{\lambda}\right\|_{L_{\left(\Sigma_{\lambda}^{u}\right)}^{k_{0}}}^{q_{3}-1}\right) \\
& \times\left\|u_{\lambda}-u\right\|_{L_{\left(\Sigma_{\lambda}^{u}\right)}^{\gamma}} \\
& +c\left(\left\|v_{\lambda}\right\|_{L_{\left(\Sigma_{\lambda}^{v}\right)}^{s_{12}}}^{p_{2}-1}+\left\|v_{\lambda}\right\|_{L_{\left(\Sigma_{\lambda}^{v}\right)}^{s_{22}}}^{q_{2}-1}+\left\|u_{\lambda}\right\|_{L_{\left(\Sigma_{\lambda}^{v}\right)}^{k_{0}}}^{q_{3}}\left\|v_{\lambda}\right\|_{L_{\left(\Sigma_{\lambda}^{v}\right)}^{p_{4}-1}}^{p_{4}-1}+\left\|u_{\lambda}\right\|_{L_{\left(\Sigma_{\lambda}^{v}\right)}^{k_{0}}}^{q_{3}}\left\|v_{\lambda}\right\|_{L_{\left(\Sigma_{\lambda}^{v}\right)}^{q_{0}}}^{q_{4}-1}\right) \\
& \times\left\|v_{\lambda}-v\right\|_{L_{\left(\Sigma_{\lambda}^{v}\right)}^{\gamma}} \\
& \leq c\left(\|u\|_{L_{\left(\left(\Sigma_{\lambda}^{u}\right)^{*}\right)}^{s_{11}}}^{p_{1}-1}+\|u\|_{L_{\left(\left(\Sigma_{\lambda}^{u}\right)^{*}\right)}^{s_{21}}}^{q_{1}-1}+\|v\|_{L_{\left(\Sigma_{\lambda}^{u}\right)}^{k_{0}}}^{p_{4}}\|u\|_{L_{\left(\left(\Sigma_{\lambda}^{u}\right) *\right)}^{k_{0}}}^{p_{3}-1}+\|v\|_{L_{\left(\Sigma_{\lambda}^{u}\right)}^{k_{0}}}^{q_{4}}\|u\|_{L_{\left(\left(\Sigma_{\lambda}^{u}\right)^{*}\right)}^{k_{0}}}^{q_{3}-1}\right) \\
& \times\left\|u_{\lambda}-u\right\|_{L_{\left(\Sigma_{\lambda}^{u}\right)}^{\gamma}}
\end{aligned}
$$

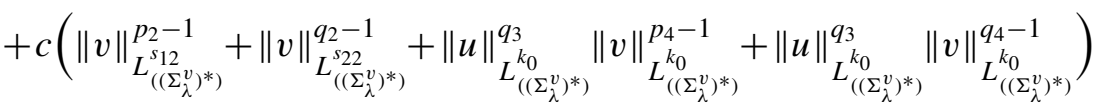

$$
\begin{aligned}
& \times\left\|v_{\lambda}-v\right\|_{L_{\left(\Sigma_{\lambda}^{v}\right)}^{\gamma}}
\end{aligned}
$$

for any $\frac{n}{n-\alpha}<\gamma<\infty$. Since $u \in \Pi_{1}, v \in \Pi_{2}$, we can choose $\varepsilon$ small enough, such that for all $\lambda \in\left[\lambda_{0}, \lambda_{0}+\varepsilon\right)$, we have

$$
\left\|u_{\lambda}-u\right\|_{L_{\left(\Sigma_{\lambda}^{u}\right)}^{\gamma}}+\left\|v_{\lambda}-v\right\|_{L_{\left(\Sigma_{\lambda}^{v}\right)}^{\gamma}} \leq \frac{1}{2}\left(\left\|u_{\lambda}-u\right\|_{L_{\left(\Sigma_{\lambda}^{u}\right)}^{\gamma}}+\left\|v_{\lambda}-v\right\|_{L_{\left(\Sigma_{\lambda}^{\nu}\right)}^{\gamma}}\right) .
$$

This implies that $\left\|u_{\lambda}-u\right\|_{L_{\left(\Sigma_{\lambda}^{u}\right)}^{\gamma}}=\left\|v_{\lambda}-v\right\|_{L_{\left(\Sigma_{\lambda}^{v}\right)}^{\gamma}}=0$. So $\Sigma_{\lambda}^{u}$ and $\Sigma_{\lambda}^{v}$ must be empty. The proof of (38) is then completed. 
Proof of Theorem 1.2. From Lemma 3.1, it follows that $u(x) \geq u_{\lambda}(x)$ and $v(x) \geq$ $v_{\lambda}(x)$ on $\Sigma_{\lambda}$ for $\lambda$ enough negative. This implies the possibility of moving the plane from near $x_{1}=-\infty$, so we can invoke Step 2: move the plane to the limiting position to derive symmetry. Furthermore, it follows from Lemma 3.2 that if the plane stops at $x_{1}=\lambda_{0}$ for some $\lambda_{0}<0$, then $u(x)$ and $v(x)$ must be symmetric and monotonic about the plane $x_{1}=\lambda_{0}$. Otherwise, we can move the plane all the way to $x_{1}=0$. Since the direction of $x_{1}$ can be chosen arbitrarily, we deduce that $u(x)$ and $v(x)$ must be radially symmetric and monotonically decreasing about some point. This completes the proof of Theorem 1.2.

\section{Classification of positive solutions to system (4) with critical exponents}

In this section, we prove Theorem 1.3. Since we have established the regularity and radial symmetry of solutions to system (4) in previous sections, we may employ a proposition in [ $\mathrm{Li}$ and Zhu 1995; Li and Zhang 2003] to show the form of radially symmetric solutions of (4) with critical exponents. Throughout this section, we always assume that $p_{1}=p_{2}=q_{1}=q_{2}=p_{3}+p_{4}=q_{3}+q_{4}=\frac{n+\alpha}{n-\alpha}$ in system (4) and $(u, v) \in L^{2 n /(n-\alpha)}\left(\mathbb{R}^{n}\right) \times L^{2 n /(n-\alpha)}\left(\mathbb{R}^{n}\right)$. It is well known that system (4) (or (1)) is invariant with respect to scaling, translation, and inversion transformations with the above exponent conditions.

For $x \in \mathbb{R}^{n}$ and $\lambda>0$, consider the Kelvin transformation of $w$ :

$$
w_{x, \lambda}(y)=\left(\frac{\lambda}{|y-x|}\right)^{n-\alpha} w\left(x+\frac{\lambda^{2}(y-x)}{|y-x|^{2}}\right) .
$$

To classify solutions, we need the following lemma.

Lemma 4.1. Let $(u, v)$ be a pair of solutions of system (4) with the assumptions of Theorem 1.3. Then there exist $\lambda>0$ and $x_{0} \in \mathbb{R}^{n}$ such that

$$
\begin{aligned}
& u_{x_{0}, \lambda}(y)=u(y), \\
& v_{x_{0}, \lambda}(y)=v(y) .
\end{aligned}
$$

Proof. It suffices to prove (40). The proof of (41) is similar. Consider $x_{0}=0$, for otherwise we make a translation transform and a scaling transform on $u_{x_{0}, \lambda}(y)$. Let $(u, v)$ be a pair of solutions of (4). By radial symmetry we assume without loss of generality that $u(x)$ and $v(x)$ are symmetric about the origin and $\lim _{|x| \rightarrow \infty}|x|^{n-\alpha} u(x)=u_{\infty}=u(0)$. Let $\lambda^{n-\alpha}=u_{\infty} / u(0)$ and $e$ be any unit vector in $\mathbb{R}^{n}$. We define

$$
w(y)=\frac{1}{|y|^{n-\alpha}} u\left(\frac{y}{|y|^{2}}-e\right) .
$$

Then

$$
w(0)=u_{\infty} \quad \text { and } \quad w(e)=u(0)
$$


Thus, $w$ must be symmetric about $\frac{1}{2} e$.

Now, choosing $y=\left(\frac{1}{2}-h\right) e$ for any $h$, as in [Chen et al. 2006], it is easy to see

$$
w\left(\left(\frac{1}{2}-h\right) e\right)=\left(\frac{1}{\left|\frac{1}{2}-h\right|}\right)^{n-\alpha} u\left(\frac{\frac{1}{2}-h}{\left|\frac{1}{2}-h\right|^{2}} e-e\right)=\left(\frac{1}{\left|\frac{1}{2}-h\right|}\right)^{n-\alpha} u\left(\frac{\frac{1}{2}+h}{\frac{1}{2}-h} e\right),
$$

where

$$
\frac{\frac{1}{2}-h}{\left|\frac{1}{2}-h\right|^{2}} e-e=e\left(\frac{\left(\frac{1}{2}-h\right)-\left(\frac{1}{2}-h\right)^{2}}{\left|\frac{1}{2}-h\right|^{2}}\right)=e \frac{\left(\frac{1}{2}-h\right)\left(1-\frac{1}{2}+h\right)}{\left(\frac{1}{2}-h\right)^{2}}=e^{\frac{1}{2}+h} \frac{1}{\frac{1}{2}-h} .
$$

Taking $y=\left(\frac{1}{2}-h\right) e$, we have

$$
w\left(\left(\frac{1}{2}+h\right) e\right)=\left(\frac{1}{\left|\frac{1}{2}+h\right|}\right)^{n-\alpha} u\left(\frac{\frac{1}{2}-h}{\frac{1}{2}+h} e\right) .
$$

Since $w$ is symmetric about $\frac{1}{2} e$, by scaling we have

$$
\frac{\lambda^{(n-\alpha) / 2}}{\left|\frac{1}{2}-h\right|^{n-\alpha}} u\left(\lambda \frac{\frac{1}{2}+h}{\frac{1}{2}-h} e\right)=\frac{\lambda^{(n-\alpha) / 2}}{\left|\frac{1}{2}+h\right|^{n-\alpha}} u\left(\lambda \frac{\frac{1}{2}-h}{\frac{1}{2}+h} e\right) .
$$

Letting $t=\left(\frac{1}{2}-h\right) /\left(\frac{1}{2}+h\right)$, it follows that

$$
u\left(\frac{\lambda e}{t}\right)=t^{n-\alpha} u(\lambda t e) .
$$

Replacing $t, e$ by $\lambda /|x-y|, y-x /|x-y|$, respectively, it follows that $u(y-x)=$ $(\lambda /|y-x|)^{n-\alpha} u\left(\lambda^{2}(y-x) /|y-x|^{2}\right)$. Furthermore, we can take the translation transform to obtain (40).

To prove our main result, we also need the following proposition from [Li and Zhang 2003]. Earlier versions with stronger assumptions were first proved by $\mathrm{Li}$ and Zhu [1995].

Proposition 4.2 [Li and Zhang 2003]. Let $f \in C^{1}\left(\mathbb{R}^{n}\right), \lambda>0$ and $\mu>0$. Suppose that for every $x \in \mathbb{R}^{n}$, there exists $\lambda(x)>0$ such that

$$
\left(\frac{\lambda}{|y-x|}\right)^{\mu} f\left(x+\frac{\lambda^{2}(y-x)}{|y-x|^{2}}\right)=f(y), \quad y \in \mathbb{R}^{n} \backslash\{x\} .
$$

Then for some $a \geq 0, d>0$ and $\bar{x} \in \mathbb{R}^{n}$, we have

$$
f(x) \equiv \pm a\left(\frac{1}{d+|x-\bar{x}|^{2}}\right)^{\mu / 2} .
$$

Proof of Theorem 1.3. From Lemma 4.1 and Proposition 4.2, we obtain directly that the solution of system (4) must be of the form (9). 


\section{Equivalence of system (1) and system (4)}

In this section, we show the equivalence of system (1) and the integral system (4). The proof is similar to that in [Chen and Li 2011] which is based on properties and the Fourier transform of the Riesz potential. For completeness and convenience of the reader, the details will be included. However, by choosing a suitable cut-off function, we provide a different approach for the case of even numbers $\alpha=2 \mathrm{~m}$.

First, we define a positive solution of (1) in the distribution sense, i.e, $u, v \in$ $H^{\alpha / 2}\left(\mathbb{R}^{n}\right)$, and they satisfy

$$
\begin{aligned}
& \int_{\mathbb{R}^{n}}(-\Delta)^{\alpha / 4} u(-\Delta)^{\alpha / 4} \phi d x=\int_{\mathbb{R}^{n}}\left(\lambda_{1} u^{p_{1}}+\mu_{1} v^{p_{2}}+\beta_{1} u^{p_{3}} v^{p_{4}}\right) \phi d x, \\
& \int_{\mathbb{R}^{n}}(-\Delta)^{\alpha / 4} v(-\Delta)^{\alpha / 4} \phi d x=\int_{\mathbb{R}^{n}}\left(\lambda_{2} u^{q_{1}}+\mu_{2} v^{q_{2}}+\beta_{2} u^{q_{3}} v^{q_{4}}\right) \phi d x
\end{aligned}
$$

for any $\phi \in C_{0}^{\infty}\left(\mathbb{R}^{n}\right)$ with $\phi(x)>0$. As usual, by the Fourier transform we have

$$
\left.\int_{\mathbb{R}^{n}}(-\Delta)^{\alpha / 4} u(-\Delta)^{\alpha / 4} \phi d x=c_{n} \int_{\mathbb{R}^{n}}|\xi|^{\alpha} \hat{u}(\xi) \phi \hat{(\xi)}\right) d \xi,
$$

where $\hat{u}$ and $\hat{\phi}$ are the Fourier transforms of $u$ and $\phi$, respectively.

For $\alpha=2 m$, where $m$ is a positive integer, we prove that every positive solution of PDE system (1) satisfies integral system (4). Here we don't use the maximum principles for higher-order elliptic operators; the method be used here comes from [Lu and Zhu 2011].

Lemma 5.1. Any positive solutions of system (1) with $\alpha=2 m$ satisfy the integral system (4).

Proof. We define the cut-off function on $B_{R}(0)$ :

$$
\eta(x)= \begin{cases}1, & x \in B_{1}(0), \\ 0, & x \notin B_{2}(0),\end{cases}
$$

and $0<\eta^{(i)}<2$ on $B_{2}(0)$ for $i=1,2, \ldots, 2 m$. Let $\eta_{R}(x-y)=\eta\left(\frac{|x-y|}{R}\right)$ on $B_{2}(x)$ and choose $\phi(x-y)=\eta_{R}(x-y) /|x-y|^{n-2 m}$. It is easy to check that $\phi \in C_{0}^{\infty}\left(\mathbb{R}^{n}\right)$. Hence, for any $u, v \in H^{m}\left(\mathbb{R}^{n}\right)$, by definition (42) and integration by parts, we have

$$
\begin{aligned}
\int_{\mathbb{R}^{n}}(-\Delta)^{m / 2} u(-\Delta)^{m / 2} \phi d y & =\int_{\mathbb{R}^{n}} u(-\Delta)^{m} \phi d y \\
& =\int_{\mathbb{R}^{n}} u(-\Delta)^{m}\left(\frac{\eta_{R}(x-y)}{|x-y|^{n-2 m}}\right) d y \\
& =\int_{\mathbb{R}^{n}} Q_{y}(u, v) \frac{\eta_{R}(x-y)}{|x-y|^{n-2 m}} d y,
\end{aligned}
$$


where $Q_{y}(u, v)$ is defined in Section 3. Since

$$
\begin{aligned}
& (-\Delta)^{m}\left(\frac{\eta_{R}(x-y)}{|x-y|^{n-2 m}}\right) \\
& \quad=(-\Delta)^{m}\left(\frac{1}{|x-y|^{n-2 m}}\right) \eta_{R}(x-y)+\sum_{i=1}^{2 m} c_{i}|x-y|^{-n+i} \eta_{R}^{(i)} R^{-i},
\end{aligned}
$$

one has

$$
\begin{array}{r}
\int_{\mathbb{R}^{n}} u(-\Delta)^{m}\left(\frac{\eta_{R}(x-y)}{|x-y|^{n-2 m}}\right) d y=\int_{\mathbb{R}^{n}} u(-\Delta)^{m}\left(\frac{1}{|x-y|^{n-2 m}}\right) \eta_{R}(x-y) d y \\
+\sum_{i=1}^{2 m} c_{i} \int_{\mathbb{R}^{n}} R^{-i} u|x-y|^{-n+i} \eta_{R}^{(i)} d y .
\end{array}
$$

As in [Lu and Zhu 2011], for $u \in L^{2 n /(n-2 m)}\left(\mathbb{R}^{n}\right)$, using the Hölder inequality we get

$$
\begin{aligned}
& \int_{\mathbb{R}^{n}} u(x-y)^{-n-i} \eta_{R}^{(i)} R^{-i} d y \\
& \leq c_{i} R^{-i}\left(\int_{\mathbb{R}^{n}} u^{2 n /(n-2 m)} d y\right)^{\frac{n-2 m}{2 n}}\left(\int_{B_{2 R} \backslash B_{R}}|x-y|^{2 n(i-n) /(n+2 m)} d y\right)^{\frac{n+2 m}{2 m}} \\
& \leq \frac{c_{i}}{R^{i}} \int_{R}^{2 R} r^{2 n(i-n) /(n+2 m)} r^{n-1} d r \rightarrow 0,
\end{aligned}
$$

as $R \rightarrow \infty$. We also note that

$$
\int_{\mathbb{R}^{n}} u(-\Delta)^{m}\left(\frac{\eta_{R}(x-y)}{|x-y|^{n-2 m}}\right) d y=\int_{\mathbb{R}^{n}} \delta(x-y) u(y) d y=u(x) .
$$

Therefore, combining (45), (46), (47) with (48), we have

$$
u(x)=\int \frac{\lambda_{1} u^{p_{1}}(y)+\mu_{1} v^{p_{2}}(y)+\beta_{1} u^{p_{3}}(y) v^{p_{4}}(y)}{|x-y|^{n-2 m}} d y .
$$

In the same way, we obtain

$$
v(x)=\int_{\mathbb{R}^{n}} \frac{\lambda_{2} u^{q_{1}}(y)+\mu_{2} v^{q_{2}}(y)+\beta_{2} u^{q_{3}}(y) v^{q_{4}}(y)}{|x-y|^{n-2 m}} d y .
$$

The proof of the lemma is completed.

Now, we consider the case that $\alpha$ is not even, that is, system (1) is equivalent to the integral system (4) for any $\alpha$.

Proof of Theorem 1.4. (i) For any $\phi \in C_{0}^{\infty}\left(\mathbb{R}^{n}\right)$, set

$$
\psi(x)=\int \frac{\phi(x)}{|x-y|^{n-\alpha}} d y,
$$


so that $(-\Delta)^{\alpha / 2} \psi=\phi$, and then $\psi \in H^{\alpha}\left(\mathbb{R}^{n}\right) \subset H^{\alpha / 2}\left(\mathbb{R}^{n}\right)$, and satisfies

$$
\int_{\mathbb{R}^{n}}(-\Delta)^{\alpha / 4} u(-\Delta)^{\alpha / 4} \psi d x=\int_{\mathbb{R}^{n}} Q_{x}(u, v) \psi(x) d x .
$$

This implies

$$
\int_{\mathbb{R}^{n}} u(-\Delta)^{\alpha / 2} \psi d x=\int_{\mathbb{R}^{n}} u \phi d x=\int_{\mathbb{R}^{n}}\left\{\int_{\mathbb{R}^{n}} \frac{Q_{y}(u, v)}{|x-y|^{n-\alpha}} d y\right\} \phi(x) d x
$$

for any nonnegative $\phi \in C_{0}^{\infty}\left(\mathbb{R}^{n}\right)$. Thus, we get

$$
u(x)=\int \frac{\lambda_{1} u^{p_{1}}(y)+\mu_{1} v^{p_{2}}(y)+\beta_{1} u^{p_{3}}(y) v^{p_{4}}(y)}{|x-y|^{n-\alpha}} d y .
$$

Similarly, we have

$$
v(x)=\int \frac{\lambda_{2} u^{q_{1}}(y)+\mu_{2} v^{q_{2}}(y)+\beta_{2} u^{q_{3}}(y) v^{q_{4}}(y)}{|x-y|^{n-\alpha}} d y .
$$

(ii) Now we show that any positive solutions of the integral system (4) satisfy system (1). Assume that $u, v \in L^{2 n /(n-2 m)}\left(\mathbb{R}^{n}\right)$ are the solutions of the integral system (4). Invoking the Fourier transform on both sides of the first equation of (4), we have

$$
\hat{u}(\xi)=c_{n}|\xi|^{-\alpha} \widehat{Q_{\xi}(u, v)} .
$$

Then

$$
|\xi|^{\alpha} \hat{u}(\xi)=c_{n} \widehat{Q_{\xi}(u, v)}(\xi) .
$$

Hence, for any $\phi \in C_{0}^{\infty}\left(\mathbb{R}^{n}\right)$, by (44) one has

$$
\begin{aligned}
\int_{\mathbb{R}^{n}}(-\Delta)^{\alpha / 4} u(-\Delta)^{\alpha / 4} \phi d x & =c_{n} \int_{\mathbb{R}^{n}}|\xi|^{\alpha} \hat{u}(\xi) \phi \hat{(\xi)} d \xi \\
& =c_{n} \int_{\mathbb{R}^{n}} \widehat{Q_{\xi}(u, v) \hat{\phi}(\xi) d \xi} \\
& =c_{n} \int_{\mathbb{R}^{n}} Q_{x}(u, v) \phi(x) d x
\end{aligned}
$$

Similarly, we have

$$
\begin{aligned}
\int_{\mathbb{R}^{n}}(-\Delta)^{\alpha / 4} v(-\Delta)^{\alpha / 4} \phi d x & =c_{n} \int_{\mathbb{R}^{n}} \widehat{K_{\xi}(u, v)}(\xi) \hat{\phi}(\xi) d \xi \\
& =c_{n} \int_{\mathbb{R}^{n}} K_{x}(u, v) \phi(x) d x .
\end{aligned}
$$

This means that $(u, v)$ is a pair of solutions of

$$
\left\{\begin{aligned}
(-\Delta)^{\alpha / 2} u & =c_{n}\left(\lambda_{1} u^{p_{1}}+\mu_{1} v^{p_{2}}+\beta_{1} u^{p_{3}} v^{p_{4}}\right), \\
(-\Delta)^{\alpha / 2} v & =c_{n}\left(\lambda_{2} u^{q_{1}}+\mu_{2} v^{q_{2}}+\beta_{2} u^{q_{3}} v^{q_{4}}\right),
\end{aligned}\right.
$$


for $x \in \mathbb{R}^{n}$, in the sense of distributions. This completes the proof of the theorem.

Now, we can combine Theorems 1.2 and 1.4 to show the nonexistence results. Proof of Theorem 1.5. It suffices to verify the condition for exponents.

(i) and (ii) Under conditions (i) and (ii), respectively, the nonexistence results have been proved in [de Figueiredo and Sirakov 2005]. Combining this with our symmetry results, we find that there exist no nontrivial positive solutions $(u, v)$ with $u \in \Pi_{1} \cap L^{\infty}\left(\mathbb{R}^{n}\right), v \in \Pi_{2} \cap L^{\infty}\left(\mathbb{R}^{n}\right)$ satisfying conditions (i) and (ii), respectively. (iii) Combining the nonexistence results of Dancer, Wei and T. Weth [2010] and our symmetry results, we conclude that there exist no nontrivial positive solutions $(u, v)$ with $u \in \Pi_{1} \cap L^{\infty}\left(\mathbb{R}^{n}\right)$ and $v \in \Pi_{2} \cap L^{\infty}\left(\mathbb{R}^{n}\right)$ with $p_{1}=q_{2}=3, p_{3}=q_{4}=1$, $p_{4}=q_{3}=2$.

\section{Acknowledgements}

We would like to thank the anonymous referees for their valuable comments and suggestions. This work was supported by the Chinese National Science Foundation for Distinguished Young Scholars (grant 10925104), National Natural Science Foundation of China (grant 11101319), Foundation of Shaanxi Province Education Department (grant 2010JK549) and Zhejiang Provincial Natural Science Foundation of China (grant Y6110118).

\section{References}

[Akhmediev and Ankiewicz 1999] N. Akhmediev and A. Ankiewicz, "Partially coherent solitons on a finite background", Phys. Rev. Lett. 82:13 (1999), 2661-2664.

[Bartsch et al. 2007] T. Bartsch, Z.-Q. Wang, and J. Wei, "Bound states for a coupled Schrödinger system”, J. Fixed Point Theory Appl. 2:2 (2007), 353-367. MR 2009i:35073 Zbl 1153.35390

[Bartsch et al. 2010] T. Bartsch, N. Dancer, and Z.-Q. Wang, "A Liouville theorem, a-priori bounds, and bifurcating branches of positive solutions for a nonlinear elliptic system", Calc. Var. Partial Differential Equations 37:3-4 (2010), 345-361. MR 2011a:35110 Zbl 1189.35074

[Busca and Sirakov 2000] J. Busca and B. Sirakov, "Symmetry results for semilinear elliptic systems in the whole space", J. Differential Equations 163:1 (2000), 41-56. MR 2001m:35100 Zbl 0952.35033

[Chen and Li 2005] W. Chen and C. Li, "Regularity of solutions for a system of integral equations", Commun. Pure Appl. Anal. 4:1 (2005), 1-8. MR 2006g:45006 Zbl 1073.45004

[Chen and $\mathrm{Li}$ 2009a] W. Chen and C. Li, "Classification of positive solutions for nonlinear differential and integral systems with critical exponents", Acta Math. Sci. Ser. B Engl. Ed. 29:4 (2009), 949-960. MR 2010i:35078 Zbl 1212.35103

[Chen and $\mathrm{Li} 2009$ b] W. Chen and C. Li, "An integral system and the Lane-Emden conjecture", Discrete Contin. Dyn. Syst. 24:4 (2009), 1167-1184. MR 2010d:35068 Zbl 1176.35067

[Chen and Li 2010] W. Chen and C. Li, Methods on nonlinear elliptic equations, AIMS Series on Differential Equations \& Dynamical Systems 4, AIMS, Springfield, MO, 2010. MR 2012k:35002 Zbl 1214.35023 
[Chen and Li 2011] W. Chen and C. Li, "Super polyharmonic property of solutions for PDE systems and its applications", preprint, 2011. arXiv 1110.2539v1

[Chen et al. 2005] W. Chen, C. Li, and B. Ou, "Classification of solutions for a system of integral equations", Comm. Partial Differential Equations 30:1-3 (2005), 59-65. MR 2006a:45007 Zbl 1073.45005

[Chen et al. 2006] W. Chen, C. Li, and B. Ou, "Classification of solutions for an integral equation", Comm. Pure Appl. Math. 59:3 (2006), 330-343. MR 2006m:45007a Zbl 1093.45001

[Dancer et al. 2010] E. N. Dancer, J. Wei, and T. Weth, "A priori bounds versus multiple existence of positive solutions for a nonlinear Schrödinger system", Ann. Inst. H. Poincaré Anal. Non Linéaire 27:3 (2010), 953-969. MR 2011d:35117 Zbl 1191.35121

[Dou et al. 2011] J. Dou, C. Qu, and Y. Han, "Symmetry and nonexistence of positive solutions to an integral system with weighted functions", Sci. China Math. 54:4 (2011), 753-768. MR 2012c:45013 Zbl 1222.45003

[Esry et al. 1997] B. D. Esry, C. H. Greene, J. P. Burke, Jr., and J. L. Bohn, "Hartree-Fock theory for double condensates", Phys. Rev. Lett. 78:19 (1997), 3594-3597.

[de Figueiredo and Sirakov 2005] D. G. de Figueiredo and B. Sirakov, "Liouville type theorems, monotonicity results and a priori bounds for positive solutions of elliptic systems", Math. Ann. 333:2 (2005), 231-260. MR 2006i:35072 Zbl 1165.35360

[Fu et al. 2009] F. Fu, L. Kong, and L. Wang, "Symplectic Euler method for nonlinear high order Schrödinger equation with a trapped term", Adv. Appl. Math. Mech. 1:5 (2009), 699-710. MR 2010j:65125

[Gidas et al. 1981] B. Gidas, W. M. Ni, and L. Nirenberg, "Symmetry of positive solutions of nonlinear elliptic equations in $\mathbf{R}^{n}$ ", pp. 369-402 in Mathematical analysis and applications, Part A, edited by L. Nachbin, Adv. in Math. Suppl. Stud. 7, Academic, New York, 1981. MR 84a:35083 Zbl 0469.35052

[Guo and Liu 2008] Y. Guo and J. Liu, "Liouville type theorems for positive solutions of elliptic system in $\mathbb{R}^{N}$, Comm. Partial Differential Equations 33:1-3 (2008), 263-284. MR 2009b:35075 Zbl 1139.35305

[Hang 2007] F. Hang, "On the integral systems related to Hardy-Littlewood-Sobolev inequality", Math. Res. Lett. 14:3 (2007), 373-383. MR 2008j:26037 Zbl 1144.26031

[Hioe 1999] F. T. Hioe, "Solitary waves for $N$ coupled nonlinear Schrödinger equations", Phys. Rev. Lett. 82:6 (1999), 1152-1155.

[Li 2004] Y. Y. Li, "Remark on some conformally invariant integral equations: The method of moving spheres”, J. Eur. Math. Soc. (JEMS) 6:2 (2004), 153-180. MR 2005e:45007 Zbl 1075.45006

[Li and Zhang 2003] Y. Li and L. Zhang, "Liouville-type theorems and Harnack-type inequalities for semilinear elliptic equations", J. Anal. Math. 90 (2003), 27-87. MR 2004i:35118 Zbl 1173.35477

[Li and Zhu 1995] Y. Li and M. Zhu, "Uniqueness theorems through the method of moving spheres", Duke Math. J. 80:2 (1995), 383-417. MR 96k:35061 Zbl 0846.35050

[Lin and Wei 2005] T.-C. Lin and J. Wei, "Ground state of $N$ coupled nonlinear Schrödinger equations in $\mathbb{R}^{n}, n \leq 3$ ", Comm. Math. Phys. 255:3 (2005), 629-653. MR 2006g:35044 Zbl 1119.35087

[Liu and Wang 2008] Z. Liu and Z.-Q. Wang, "Multiple bound states of nonlinear Schrödinger systems”, Comm. Math. Phys. 282:3 (2008), 721-731. MR 2009k:58022 Zbl 1156.35093

[Liu et al. 2006] J. Liu, Y. Guo, and Y. Zhang, "Liouville-type theorems for polyharmonic systems in $\mathbb{R}^{N}$, J. Differential Equations 225:2 (2006), 685-709. MR 2007d:35092 Zbl 1147.35316 
[Liu et al. 2007] X.-S. Liu, Y.-Y. Qi, J.-F. He, and P.-Z. Ding, "Recent progress in symplectic algorithms for use in quantum systems", Commun. Comput. Phys. 2:1 (2007), 1-53. MR 2008a:81002

[Lu and Zhu 2011] G. Lu and J. Zhu, "Symmetry and regularity of extremals of an integral equation related to the Hardy-Sobolev inequality", Calc. Var. Partial Differential Equations 42:3-4 (2011), 563-577. Zbl 1231.35290

[Ma et al. 2011] C. Ma, W. Chen, and C. Li, "Regularity of solutions for an integral system of Wolff type”, Adv. Math. 226:3 (2011), 2676-2699. MR 2011k:45009 Zbl 1209.45006

[Maia et al. 2006] L. A. Maia, E. Montefusco, and B. Pellacci, "Positive solutions for a weakly coupled nonlinear Schrödinger system”, J. Differential Equations 229:2 (2006), 743-767. MR 2007h:35070 Zbl 1104.35053

[Sirakov 2007] B. Sirakov, "Least energy solitary waves for a system of nonlinear Schrödinger equations in $\mathbb{R}^{n} "$, Comm. Math. Phys. 271:1 (2007), 199-221. MR 2007k:35477 Zbl 1147.35098

[Wei and Weth 2007] J. Wei and T. Weth, "Nonradial symmetric bound states for a system of coupled Schrödinger equations", Rend. Lincei Mat. Appl. 18:3 (2007), 279-293. MR 2008g:35078 Zbl 1229.35019

[Wei and Weth 2008] J. Wei and T. Weth, "Radial solutions and phase separation in a system of two coupled Schrödinger equations", Arch. Ration. Mech. Anal. 190:1 (2008), 83-106. MR 2009k:35059 Zbl 1161.35051

[Wei and Xu 1999] J. Wei and X. Xu, "Classification of solutions of higher order conformally invariant equations”, Math. Ann. 313:2 (1999), 207-228. MR 2000a:58093 Zbl 0940.35082

[Zhang 2007] Y. Zhang, "A Liouville type theorem for polyharmonic elliptic systems", J. Math. Anal. Appl. 326:1 (2007), 677-690. MR 2007g:35070 Zbl 1142.35020

Received July 22, 2011. Revised September 7, 2012.

\section{JINGBO DOU}

SCHOOL OF STATISTICS

XI'AN UNIVERSITY OF FINANCE AND ECONOMICS

710100 XI'AN

CHINA

jbdou@xaufe.edu.cn

ChAngZheng QU

Center for Nonlinear Studies

NINGBO UNIVERSITY

315211 NINGBO

CHINA

quchangzheng@nbu.edu.cn 


\title{
PACIFIC JOURNAL OF MATHEMATICS
}

\author{
msp.org/pjm
}

Founded in 1951 by E. F. Beckenbach (1906-1982) and F. Wolf (1904-1989)

\section{EDITORS}

V. S. Varadarajan (Managing Editor)

Department of Mathematics

University of California

Los Angeles, CA 90095-1555

pacific@math.ucla.edu

Paul Balmer

Department of Mathematics

University of California

Los Angeles, CA 90095-1555

balmer@math.ucla.edu

Daryl Cooper

Department of Mathematics

University of California

Santa Barbara, CA 93106-3080 cooper@math.ucsb.edu

Jiang-Hua $\mathrm{Lu}$

Department of Mathematics

The University of Hong Kong

Pokfulam Rd., Hong Kong jhlu@maths.hku.hk
Don Blasius

Department of Mathematics University of California

Los Angeles, CA 90095-1555

blasius@math.ucla.edu

Robert Finn

Department of Mathematics Stanford University

Stanford, CA 94305-2125

finn@math.stanford.edu

Sorin Popa

Department of Mathematics

University of California

Los Angeles, CA 90095-1555

popa@math.ucla.edu

Paul Yang

Department of Mathematics

Princeton University

Princeton NJ 08544-1000

yang@math.princeton.edu

\section{PRODUCTION}

Silvio Levy, Scientific Editor, production@msp.org

\section{SUPPORTING INSTITUTIONS}

ACADEMIA SINICA, TAIPEI

CALIFORNIA INST. OF TECHNOLOGY

INST. DE MATEMÁTICA PURA E APLICADA

KEIO UNIVERSITY

MATH. SCIENCES RESEARCH INSTITUTE

NEW MEXICO STATE UNIV.

OREGON STATE UNIV.

\author{
STANFORD UNIVERSITY \\ UNIV. OF BRITISH COLUMBIA \\ UNIV. OF CALIFORNIA, BERKELEY \\ UNIV. OF CALIFORNIA, DAVIS \\ UNIV. OF CALIFORNIA, LOS ANGELES \\ UNIV. OF CALIFORNIA, RIVERSIDE \\ UNIV. OF CALIFORNIA, SAN DIEGO \\ UNIV. OF CALIF., SANTA BARBARA
}

\author{
Vyjayanthi Chari \\ Department of Mathematics \\ University of California \\ Riverside, CA 92521-0135 \\ chari@math.ucr.edu \\ Kefeng Liu \\ Department of Mathematics \\ University of California \\ Los Angeles, CA 90095-1555 \\ liu@math.ucla.edu \\ Jie Qing \\ Department of Mathematics \\ University of California \\ Santa Cruz, CA 95064 \\ qing@cats.ucsc.edu
}

These supporting institutions contribute to the cost of publication of this Journal, but they are not owners or publishers and have no responsibility for its contents or policies.

See inside back cover or msp.org/pjm for submission instructions.

The subscription price for 2013 is US \$400/year for the electronic version, and \$485/year for print and electronic.

Subscriptions, requests for back issues and changes of subscribers address should be sent to Pacific Journal of Mathematics, P.O. Box 4163, Berkeley, CA 94704-0163, U.S.A. The Pacific Journal of Mathematics is indexed by Mathematical Reviews, Zentralblatt MATH, PASCAL CNRS Index, Referativnyi Zhurnal, Current Mathematical Publications and the Science Citation Index.

The Pacific Journal of Mathematics (ISSN 0030-8730) at the University of California, c/o Department of Mathematics, 798 Evans Hall \#3840, Berkeley, CA 94720-3840, is published monthly except July and August. Periodical rate postage paid at Berkeley, CA 94704, and additional mailing offices. POSTMASTER: send address changes to Pacific Journal of Mathematics, P.O. Box 4163, Berkeley, CA 94704-0163.

PJM peer review and production are managed by EditFLOW ${ }^{\circledR}$ from Mathematical Sciences Publishers.

PUBLISHED BY

mathematical sciences publishers

nonprofit scientific publishing

http://msp.org/

(C) 2013 Mathematical Sciences Publishers 


\section{PACIFIC JOURNAL OF MATHEMATICS}

Volume $261 \quad$ No. $2 \quad$ February 2013

Geography of simply connected nonspin symplectic 4-manifolds with positive signature

Anar Akhmedov, Mark C. Hughes and B. Doug Park

Schur-Horn theorems in $\mathrm{II}_{\infty}$-factors

283

MARTÍN ARGERAMI and PEDRo MASSEY

Classification of positive solutions for an elliptic system with a higher-order

fractional Laplacian

JiNGBo DOU and CHANGZHENG QU

Bound states of asymptotically linear Schrödinger equations with compactly supported potentials

MingWEN FEI and HUICHENG YiN

Type I almost homogeneous manifolds of cohomogeneity one, III

DANIEL GUAN

The subrepresentation theorem for automorphic representations

MARCELA HANZER

Variational characterizations of the total scalar curvature and eigenvalues of the

Laplacian

Seungsu Hwang, JeOngwook Chang and GabJin Yun

Fill-ins of nonnegative scalar curvature, static metrics, and quasi-local mass

JEFFREY L. JAUREGUI

Operator algebras and conjugacy problem for the pseudo-Anosov automorphisms of a surface

\section{IGOR NIKOLAEV}

Connected sums of closed Riemannian manifolds and fourth-order conformal invariants

DAVID RASKE

Ruled minimal surfaces in the three-dimensional Heisenberg group

Heayong Shin, Young Wook Kim, Sung-Eun Koh, Hyung Yong LeE and SEONG-DEOG YANG

$G$-bundles over elliptic curves for non-simply laced Lie groups and configurations of lines in rational surfaces

MANG XU and JIAJIN ZHANG 